GA-A21236

UC-406

\title{
USER'S MANUAL FOR THE DAD-1 DATA ACQUISITION DAUGHTER BOARD FOR THE SUPERCARD-2
}

\author{
by \\ J.R. FERRON
}

Work prepared under

Department of Energy

Contract DE-AC03-89ER51114

GENERAL ATOMICS PROJECT 3466 DATE PUBLISHED: MAY 1993

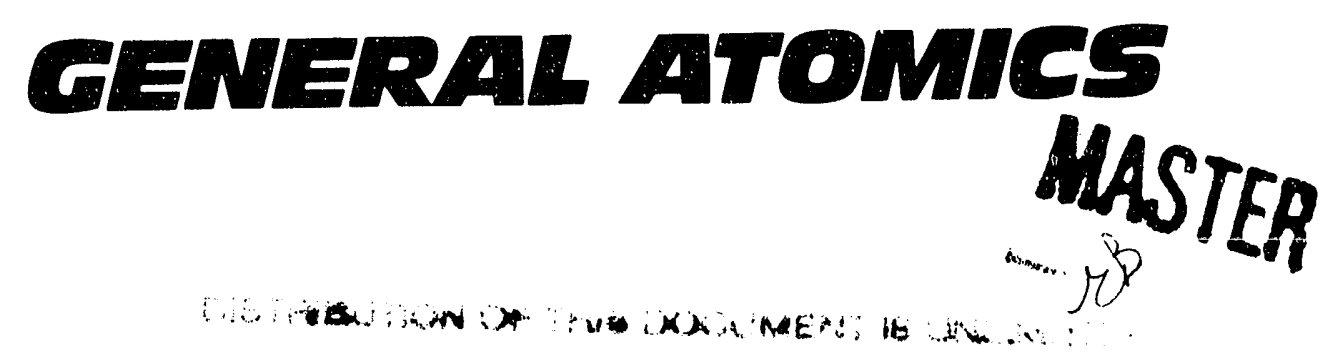




\title{
User's Manual for the DAD-1 Data Acquisition Daughter Board for the SuperCard-2*
}

\author{
J.R. Ferron \\ General Atomics \\ San Diego, California 92186-9784, U.S.A.
}

\begin{abstract}
A detailed description of how to use the DAD-1 data acquisition daughter board is given. The DAD-1 daughter board is used with the SuperCard-2, a VME format processor board manufactured by CSP Inc. that is based on the Intel i860 microprocessor. The daughter board provides high speed acquisition of digital data through a general purpose input port. Data are transferred through direct memory access operations to the memory on the SuperCard-2 board at a rate up to 40 million, 14 bit samples per second. A first-in, first-cut memory is used to buffer the data during the transfer. Several different data acquisition operating modes are available that make a combination of a SuperCard-2 processor board and a DAD-1 daughter board suitable for a wide range of real time data analysis and feedback control functions.
\end{abstract}

*This is a report of work sponsored by the U.S. Department of Ene: gy under Contract No. DE-AC03-89ER51114. 


\section{Contents}

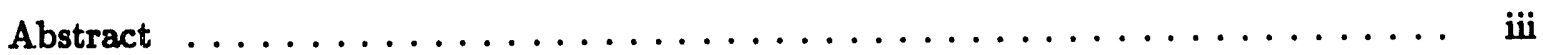

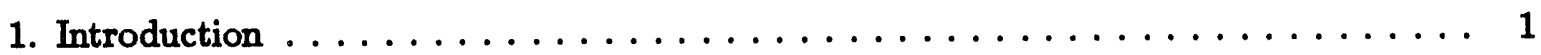

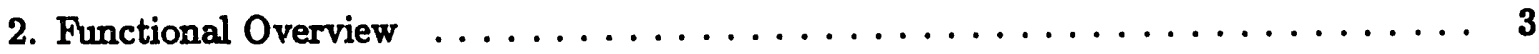

2.1. System Configuration . . . . . . . . . . . . . . . . . 3

2.2. Data Acquisition Functions Versus Control Functions . . . . . . . . . . 4

2.3. The Function of the Daughter Board $\ldots \ldots \ldots \ldots \ldots \ldots \ldots$

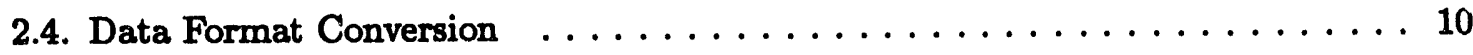

2.5. Arbitration of Access to SuperCard-2 Memory . . . . . . . . . . . . . . 12

2.6. The 8660 Cache and Data Acquisition . . . . . . . . . . . . . 14

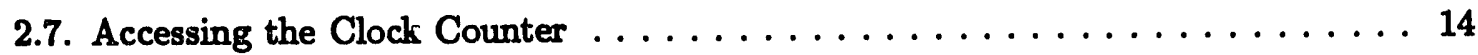

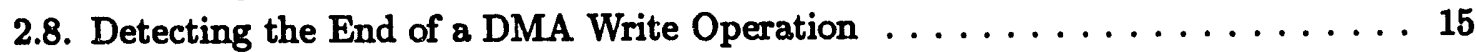

2.9. Beharior When There Is No Digital Data Source $\ldots \ldots \ldots \ldots \ldots \ldots$

2.10. SuperCard-2 Memory Page Boundaries . . . . . . . . . . . . . 16

3. Programming for the FIFO Memory Daughter Board $\ldots \ldots \ldots \ldots \ldots \ldots$

3.1. Addressing the Data Acquisition System . . . . . . . . . . . . 17

3.2. The Control Register . . . . . . . . . . . . . . . . . . 18

3.3. The Control and Status Register Number $2 \ldots \ldots \ldots \ldots \ldots$

3.4. The Clock Counter Value Register . . . . . . . . . . . . . . . 24

3.5. The Data Buffer Address Register $\ldots \ldots \ldots \ldots \ldots \ldots \ldots \ldots \ldots \ldots$

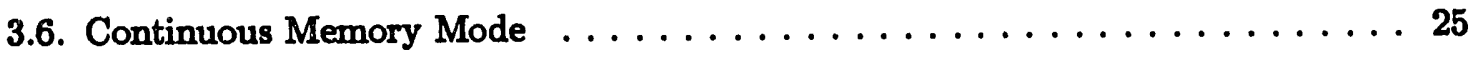

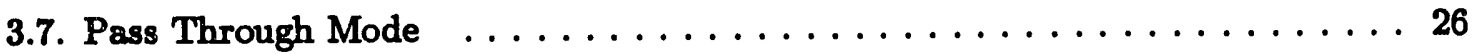

3.8. Ordering of Data in the SuperCard-2 Input Memory Buffer . . . . . . . . . 27

3.9. Power Up Status . . . . . . . . . . . . . . . . . . 30

4. Outlines of Data Acquisition Programs . . . . . . . . . . . . . 31

4.1. A Typical Feedback Control Application . . . . . . . . . . . . 31

4.2. A Typical Data Reduction Application $\ldots \ldots \ldots \ldots \ldots \ldots \ldots \ldots \ldots$

4.3. A Near Real Time Analysis Application $\ldots \ldots \ldots \ldots \ldots \ldots \ldots \ldots \ldots \ldots \ldots \ldots$

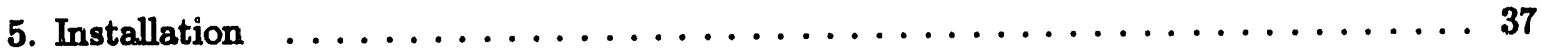

6. Acknowledgments $\ldots \ldots \ldots \ldots \ldots \ldots \ldots \ldots \ldots \ldots \ldots \ldots \ldots \ldots \ldots \ldots \ldots \ldots$ 


\section{Figures}

1. Simplified block diagram of the FIFO memory daughter board . . . . . . . . . 5

2. Block diagram of a complete data acquisition and processing system . . . . . . 6

3. Data transfer from the FIFO memory to SuperCard-2 memory . . . . . . . . . . 11

4. Data paths on the SuperCard-2 and FIFO memory daughter board . . . . . . . 13

5. Order of data in the DMA memory buffer . . . . . . . . . . . . . . 29

6. Attaching the FIFO meniory daughter board to the SuperCard-2 . . . . . . . . 38

\section{Tables}

1. Specifications for the FIFO memory data acquisition daughter board $\ldots \ldots \ldots \ldots$

2. The FIFO memory daughter board registers $\ldots \ldots \ldots \ldots \ldots \ldots \ldots$

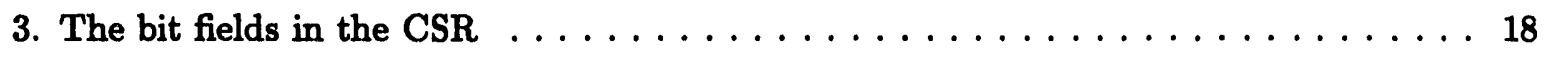

4. The bit fields in the CSR2 register $\ldots \ldots \ldots \ldots \ldots \ldots \ldots \ldots \ldots$ 


\section{Introduction}

This document describes how to use the first-in, first-out (FIFO) memory data acquisition daughter board for the SuperCard-2. This manual covers only the daughter board; the sources of input data are covered in separate manuals. The information required for the application programmer is given here, while detailed descriptions of the circuit operation are given in separate manuals.

The first section gives an overview of how the FIFO memory daughter board can be used for data acquisition and how it fits into a high speed data acquisition system. The second section is a detailed description of the programmer's view of the FIFO memory daughter board and the third section outlines some application examples. Finally, the last section covers installation of the FIFO memory daughter board. 


\section{Functional Overview}

This section gives an overview of the capabilities of the FIFO memory daughter board and how it fits into a complete data acquisition and analysis system. The daughter board specifications are summarized in Table 1.

Table 1

Specifications for the FIFO Memory Data Acquisition Daughter Board

Format:

VME interface:

Space requirement:

Power:

Data input rate:

Digital input data width:

Rate of DMA transfer to SuperCard-2 memory:

Maximum "clock counter" clock rate

\section{U VME}

norie, only power and ground connections are used

1 VME slot on the right-hand side of the SuperCard-2

\section{$5 \mathrm{~A}$ at $5 \mathrm{~V}$}

$5 \mathrm{MHz}$ maximum on each of eight parallel buses

14 bits on each of eight buses 40 Msamples/sec in bursts of fewer than 308 samples

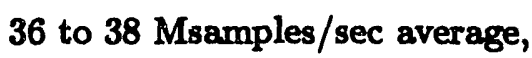
sustained

$5 \mathrm{MHz}$

\subsection{System Configuration}

The purpose of the FIFO memory daughter board is to accept digital data from some type of external data source and transfer the data to the memory on the SuperCard-2 processor board through direct memory access (DMA) operations. There is flexibility in how this is done so that the combination of the FIFO memory daughter board and the SuperCard-2 can be adapted to a wide variety of data acquisition and data analysis applications. The data input interface to the daughter board is designed to be easily connected to a variety of data sources. 
Figure 1 shows a block diagram of the FIFO memory daughter board. There are eight input data buses, each 14 bits wide, which are connected to the external source of digital data. These buses provide data to two input multiplexers, each of which accepts four digital input values of up to 14 bits each in parallel at an arbitrary rate up to $5 \mathrm{MHz}$. Each multiplexer transfers the data to a separate FIFO memory at a $20 \mathrm{MHz}$ rate. DMA operations are used to transfer the data in parallel from the two FIFO memories to the SuperCard-2 memory, also at a $20 \mathrm{MHz}$ rate, resulting in a maximum possible data acquisition speed of 40 million values per second.

Figure 2 shows how the FIFO memory daughter board fits into a complete data acquisition system. The example here shows commercially available digitizers in use as the digital data source, but a wide variety of data sources can be easily interfaced to the FIFO memory board. The real time processor board, a SuperCard-2 manufactured by CSP Inc., has four basic elements: an Intel 80860 (also called i860) microprocessor, 16 Mbytes of memory, a VME bus interface, and a 64 bit wide daughter board port. The daughter board port is designed to allow customers to build custom interface boards such as the FIFO memory board. The VME interface is used for communication with the host computer and can also be used to communicate with other VME format circuits for analog and digital input and output.

Each SuperCard-2 in the system is coupled with a FIFO memory daughter board. As shown in the figure, there can be multiple daughter/processor board combinations. The input data are provided to all of the FIFO memory boards in parallel allowing for parallel acquisition and processing of data.

\subsection{Data Acquisition Functions Versus Control Functions}

The combination of digital data source, daughter board, SuperCard-2 and host computer makes a general purpose high speed data acquisition and analysis system. Setup options are available which give the system operating modes for three basic types of applications.

1. In a feedback control application, it is necessary that the data be acquired, processed and a command output be produced with as little delay as possible between the data acquisition and output. In this case the data pass through the FIFO memory as quickly as possible.

2. In a data acquisition and real time data reduction application, data are acquired and processed and the processed result is stored in memory. In this case, the output of the data reduction calculation is not used as a feedback command so that it is only necessary that the data processing proceeds on average as quickly the data acquisition. The input data can be held in the FIFO memory or a SuperCard-2 memory buffer until the processor is ready.

3. The memory on the SuperCard-2 can be simply used for data storage during a pulse of an experiment. The data are then analyzed in "near real time," that is, as soon after 


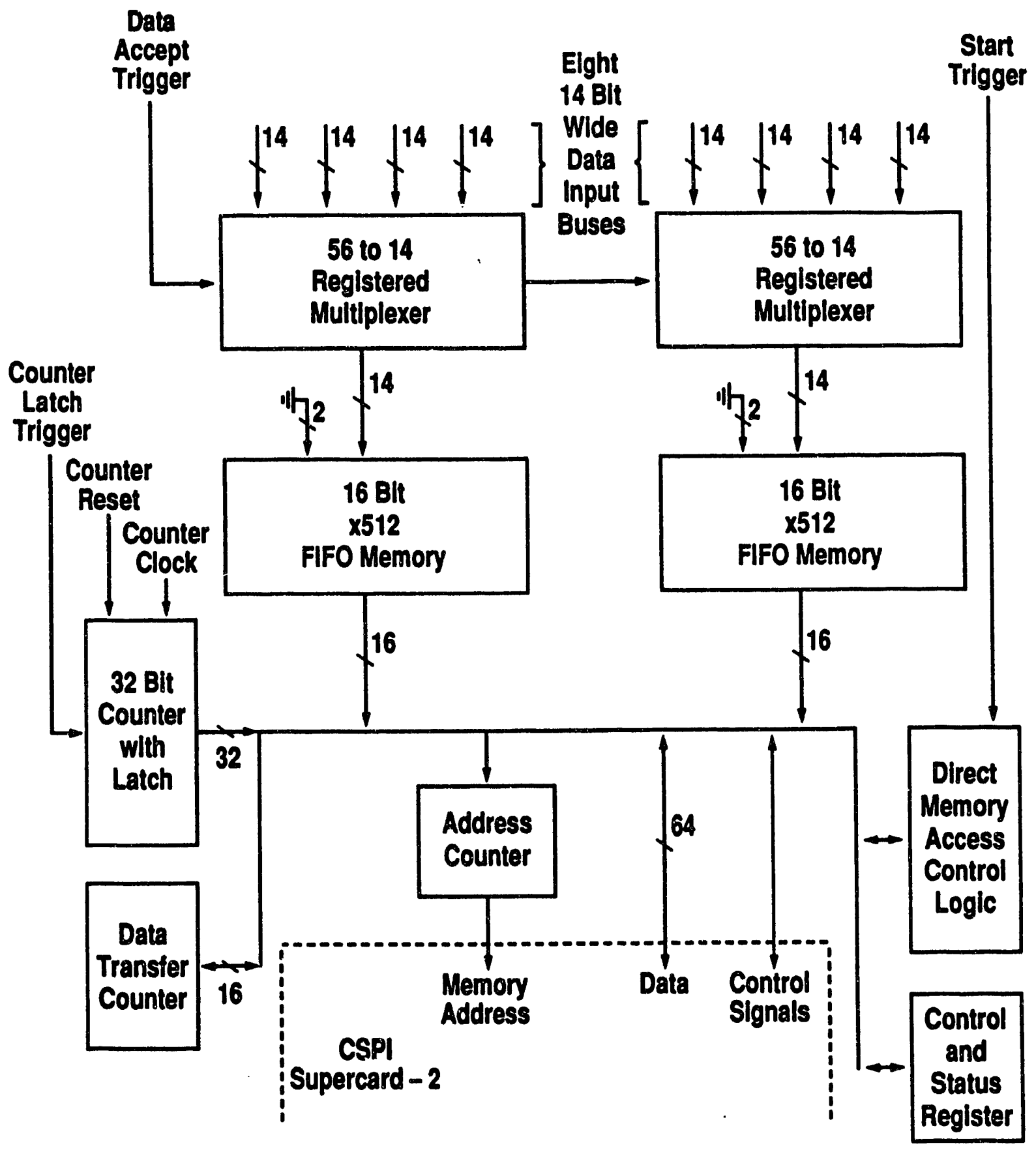

Fig. 1. Simplified block diagram of the FIFO memory daughter board. 


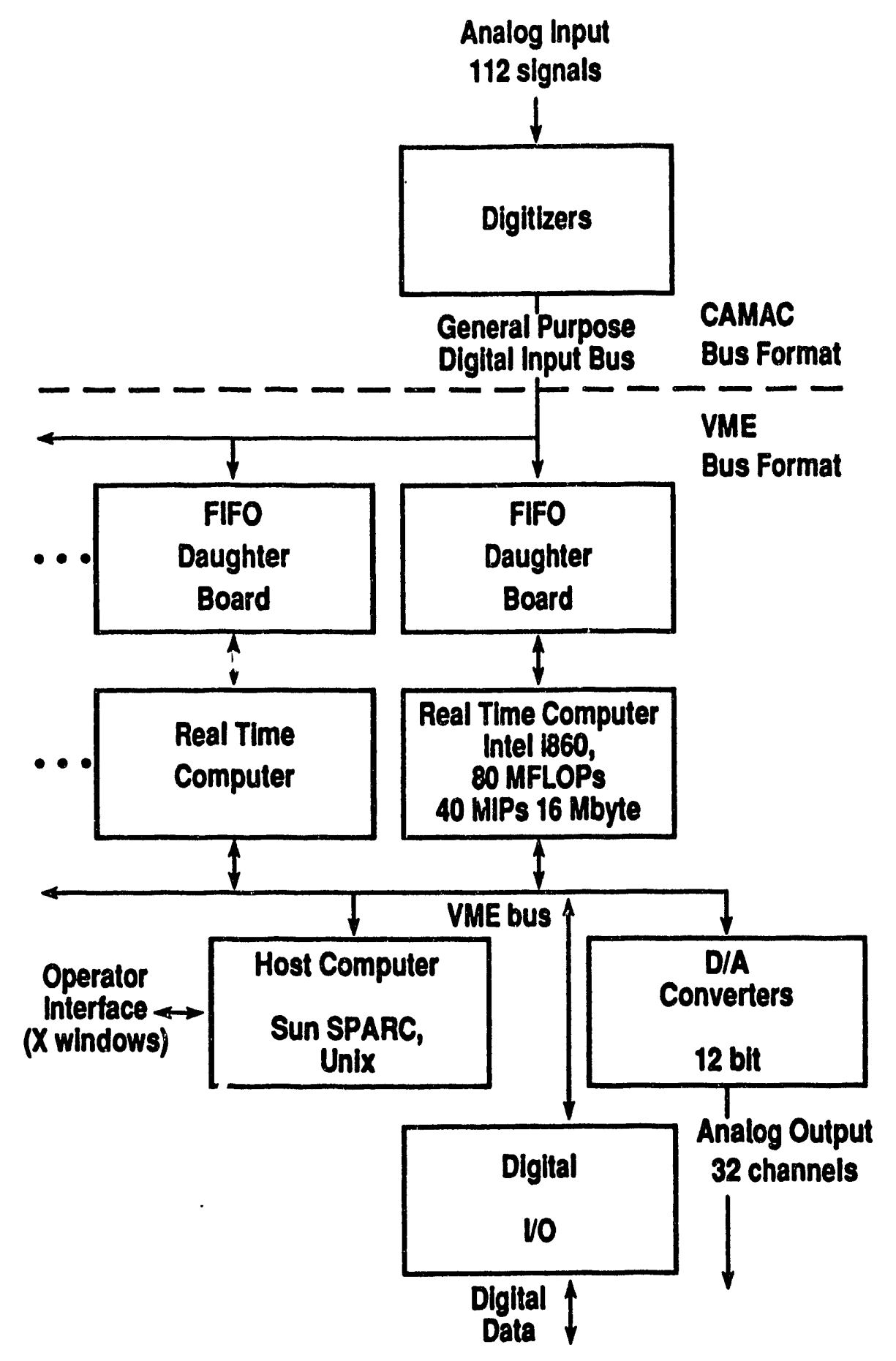

FiG. 2. Block diagram of a complete data acquisition and processing system. 
the experiment pulse as possible. This is useful when there is no special requirement for data reduction or when the data reduction algorithm is too slow to be executed in real time. In this case the "continuous memory mode" described in Section 4 can be used to optimize the use of the SuperCard-2 memory.

In Section 4, suggested daughter board setups are given for each of these modes of operation.

\subsection{The Function of the Daughter Board}

The first-in, first-out memory daughter board provides the interface between an external digital data source and the SuperCard-2. The daughter board performs only two basic functions: it can accept requests for access by the i860 processor to one of its on board registers or it can execute a DMA write cycle to transfer data from the external data source to the SuperCard-2 memory.

The daughter board has a general purpose data input port which is designed to accommodate a variety of types of digital data sources. The control interface from the data source to the FIFO memory board is simple, utilizing only 2 primary trigger signals, "data accept trigger" and "start trigger." The data accept trigger is a signal to the daughter board that there are data available on the input bus and that these data should be copied into the FIFO memory. One of the functions of the start trigger is to signal the daughter board to start a DMA operation. See the separate manual, "Input Interface Design Guide for the DAD-1 Data Acquisition Daughter Board for the SuperCard-2," for details on connecting a source of data to the FIFO memory daughter board.

The data that come through the input port are first placed into a pair of 16 bit wide by 512 deep FIFO memories. Data are copied to the FIFO memory from the data input port on each cycle of the data accept trigger. The FIFO memory serves as a buffer between the digital data source and the SuperCard-2 so that there is no requirement for synchronization of timing in the two systems. Also, it is possible to daisy-chain several daughter board/SuperCard-2 combinations so that the same data can be provided simultaneously to more than one processor for parallel processing applications (as illustrated in Fig. 2). The FIFO memory also eliminates any requirement of close synchronization between the several SuperCard-2 processor boards in this case.

In response to one of several possible triggers, the daughter board will request access to the SuperCard-2 memory and copy the data from the FIFO memory into the SuperCard-2 memory. The various options for daughter board behavior are chosen by programming the board's two control and status registers. 
There are three ways that a transfer of data from the FIFO memory into the SuperCard-2 memory can be triggered: (a) a logic 1 on the start trigger signal can start a transfer, (b) a transfer can be started automatically when more than one-half of the available FIFO memory is filled, or (c) the i860 processor can request the immediate start of a transfer. There are three ways to end a data transfer: (a) when the FIFO memory becomes empty and the start trigger signal is at logic 0 , (b) when a specified number of data values has been transferred from the FIFO memory, or (c) when the FIFO memory is empty.

Start method (a) and stop method (a) are designed to be used together when the data acquisition is controlled primarily by the external data source. The external data source signals a request for a DMA transfer with the start trigger signal immediately before it begins the transfer of data to the FIFO memory. Data are then transferred continuously from the FIFO memory to the SuperCard-2 memory. The FIFO memory may become temporarily empty if the external source does not provide data quickly enough. In this case, the direct memory access operation simply pauses until data are again available in the FIFO memory. In this scheme, there is the shortest possible delay in the transfer of the data from the external source to the SuperCard-2 memory, a situation well suited to a real time feedback control application. When the external source has finished providing data, the start trigger signal is removed, but the DMA transfer does not end until the FIFO memory empties so that all available data are written to the processor memory.

Start method (b) and stop method (b) are designed to be used together when the external source provides a continuous stream of data at an arbitrary rate and the 1860 processes the data in batches. In this case the 1860 processor controls the data transfer by requesting a specific number of data values in each DMA transfer from the FIFO memory to the SuperCard-2 memory. The external source controls the transfer of data into the FIFO memory. This setup is suited to applications where a delay between the sampling of the data and processing of the data can be tolerated, such as real time reduction and/or storage of the input data where control feedback is not necessary. When the FIFO memory is more than half full a direct memory access transfer is automatically triggered and, when the required number of values has been written to the SuperCard-2 memory, the transfer ends. The i860 then processes this batch of data while the next batch is written to the FIFO memory. The desired amount of data in each batch is specified by a value written by the $i 860$ into the data transfer counter.

Any combination of data transfer start and stop methods can be programmed so that the data acquisition protocol can be chosen to match a wide variety of applications. The ability to read the data transfer counter to determine the number of data values transferred enhances this flexibility. The data transfer activity is enabled or disabled through control bits in the control and status register. This register can also be used to clear the content of 'the FIFO memory, provide trigger and enable signals to the external data source, or reset the daughter board and the external data source. 
The location of the SuperCard-2 memory buffer that receives the data is controlled by a value written by the $i 860$ to the address register. At the start of a DMA cycle, the value in the address register is (optionally) copied to the address counter which provides the actual memory address to the SuperCard-2 circuits. In addition, the address counter is loaded each time the address register is loaded. The address counter is incremented after each write of data to SuperCard-2 memory so that it then points to the next memory location to receive data.

There are several options which affect the behavior of the address counter. First, at the start of each DMA cycle, the address counter can be loaded from the address register. In this mode, the same block of SuperCard-2 memory receives the input data on each DMA cycle unless the value in the address register is changed. This mode is useful if the data are to be processed in some manner and then discarded. In the second mode of operation, the address counter is loaded only when the i860 processor loads the address register. Consecutive DMA operations will then fill consecutive blocks in the SuperCard-2 memory because the address counter will always increment to point to the next location in memory to receive data and the address counter will not be altered between DMA cycles.

A separate mode of operation is called "continuous memory" mode (see Subsection 3.6 for details). As discussed in Subsection 2.4, only 32 of the 64 bits in the daughter board interface port are actually used during each write cycle and the memory which maps to the other 32 bits is not altered. When the address counter is incremented after every memory write operation, only half of the memory is actually written. In continuous memory mode, the address counter increments after every other write operation and on alternating write operations, alternating halves of the 64 bit bus are used. In this manner, every byte of tine SuperCard-2 memory can be written. Continuous memory mode can be used with either of the address counter load modes described in the previous paragraph.

A 32 bit counter with an associated latch is used to provide timing information. The counter is designed to be cleared by a "counter reset" signal and incremented by a "counter clock" in order to record the time, or count some set of events, in the experiment. The counter clock can have any frequency up to $5 \mathrm{MHz}$. The value of the counter can be latched at the time indicated by the "counter latch trigger." These signals are provided by the external source of data. The latched value can be read directly by the $i 860$ processor. Also, the latched value is (optionally) written to SuperCard-2 memory at the address which directly follows the last data copied from the FIFO memory during a DMA write cycle. Thus a block of data can be automatically labeled with the value from the counter, which might be, for instance, the trigger time of the set of data samples.

In some data processing algorithms, particularly in a multiprocessor setup, it may be desirable for the $i 860$ processor to ignore some of the input data. Because the processor cannot access the SuperCard-2 memory during a DMA operation by the FIFO memory daughter board (see Subsection 2.5), data to be ignored should not be written to SuperCard-2 memory so that an unnecessary DMA operation does not slow the processing algorithm. In this 
situation, the "pass through" mode of operation can be used. In this mode, the daughter board behaves in the same way as in a normal DMA operation except that no data are actually written to the SuperCard-2 memory. Thus the data passing through the FIFO memory during a pass through DMA operation is effectively ignored. However, the quantity of data to be ignored can be easily controlled by the processor.

\subsection{Data Format Conversion}

A typical digital data source will provide integer data. The i860 microprocessor operates most effectively on floating point values. Thus the task of acquiring the digital data and providing the results to the microprocessor usually must include the conversion to floating point. This section describes how the conversion from integer to floating point format is accommodated by the design of the data acquisition circuit.

In the conversion algorithm the integer is placed justified toward the least significant side in the least significant 23 bits of a floating point register (with zero written to any of the 23 bits which are not defined by the input data) and the upper 9 bits of the register are set to a fixed mask value as shown in Fig. 3(a). This results in a valid IEEE format 32 bit floating point value which equals the integer value plus $2^{23}$. From this value a floating point value equal to $2^{23}$ is subtracted, resulting in a properly normalized floating point value equal to the integer input value.

The daughter board supports this algorithm by writing the integer data to memory in a manner so that no extra processing is required in order to add the mask value. The daughter board circuit transfers two data values simultaneously to SuperCard-2 memory, one in each 32 bit half of the 64 bit input data bus. The conversion algorithm requires that the data be placed in one half of the 32 bits and the appropriate mask in the other half. The daughter board circuit actually only transfers the data using two 16 bit sections of the data bus. The memory bytes connected to the other 16 bit sections are not altered. The required mask value is placed in the memory buffer by the 1860 processor and the daughter board writes data only in the appropriate bytes. This is illustrated in Fig. 3(b).

The data acquisition design described here which accommodates the integer to floating point conversion algorithm results in only every other pair of bytes being written with data. This is not desirable when the memory is used for storage and near real time processing is performed. It that case every byte of memory should be used. There is a provision, called continuous memory mode, to have data written into every memory byte. In this case it is assumed that a lower speed integer to floating conversion algorithm can be used and that best utilization of memory has the highest priority. 
(a)

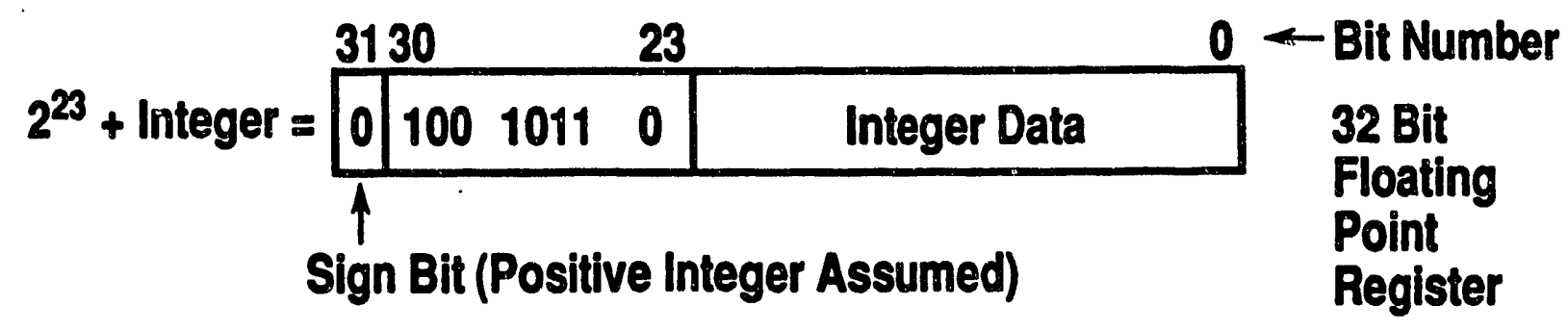

(b)
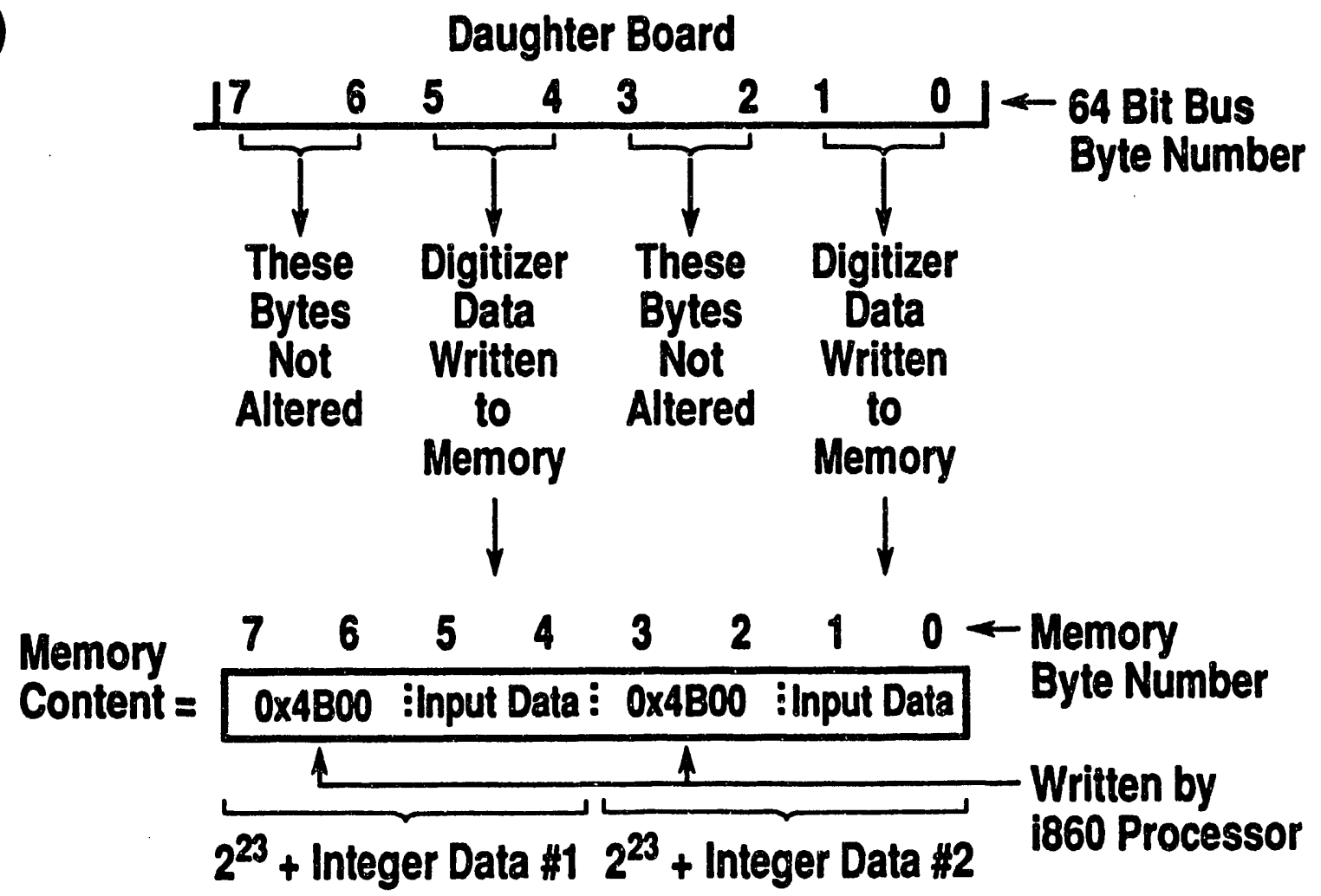

Fig. 3. (a) Bit arrangement for conversion from integer to floating point,

(b) Data flow from the FIFO memory daughter board to SuperCard-2 memory. 


\subsection{Arbitration of Access to SuperCard-2 Memory}

The i860 processor cannot access the random access memory while the DMA transfer of data from the FIFO memory is in progress becuuse, as shown in Fig. 4, there is a common data path between the FIFO memory, the random access memory, and the 1860 processor. Therefore, the amount of time available for memory access during data processing depends on how close the average rate of data transfer is to the maximum transfer rate and the extent to which the i860 processor's cache memory can be used during the analysis. At the maximum, $40 \mathrm{Msample} / \mathrm{sec}$, data transfer rate the common dat s path is fully utilized by the data transfer process and it is not possible for the $\mathrm{i} 860$ processor to access the random access memory. Also, the i860 processor cannot access the various registers on the FIFO memory daughter board while there is a DMA transfer in progress.

The SuperCard-2 circuits arbitrate between requests for access to the daughter board registers and the SuperCard-2 random access memory, assigning the VME interface the highest priority, followed by the daughter board and then the i860 processor. The absolute highest priority is assigned to requests for refresh of the dynamic random access memory on the SuperCard-2. Once access to memory or a daughter board register has been granted, however, the access continues without interruption until completion.

This arbitration affects the timing of both data transfers and access to the daughter board registers. If a DMA data transfer is requested by the daughter board and a memory refresh or an access to SuperCard-2 memory by the i860 processor or the VME bus is in progress, the daughter board waits until access to memory is granted. Thus there can be some uncertainty in the exact time of the start of a DMA data transfer. If the i860 processor requests access to a daughter board register while a DMA transfer is in progress, the i860 processor is forced to wait.

During a DMA transfer from the daughter board to SuperCard-2 memory, the daughter board does not access the memory for a period longer than $8 \mu \mathrm{sec}$. This prevents problems which could occur if the memory refresh does not occur often enough or if there was too long a delay after a request by a VME device to accers the SuperCard-2 memory. If the amount of data to be transferred is large enough that the DMA operation requires more than $8 \mu \mathrm{sec}$, the DMA transfer must pause briefly and then start again. During the pause, at the very least a memory refresh could occur; this is not under the control of the application programmer. There could also be access to memory by the i 860 processor or a VME device, but the possibility of this type of access is under the control of the programmer.

Thus, continuous trarsters of data from the daughter board to SuperCard-2 memory cannot occur at the maximum rate of 40 million samples per second. The maximum rate can only be sustained in bursts of less than $8 \mu \mathrm{sec}$ in duration. A maximum of 308 data values can be transferred from the FIFO memory in an $8 \mu \mathrm{sec}$ burst (154 values on each half of the 64 bit daughter board bus). Assuming that the programmer has ensured that memory refresh is the only interfering process, the maximum sustained data transfer rate (on average over 


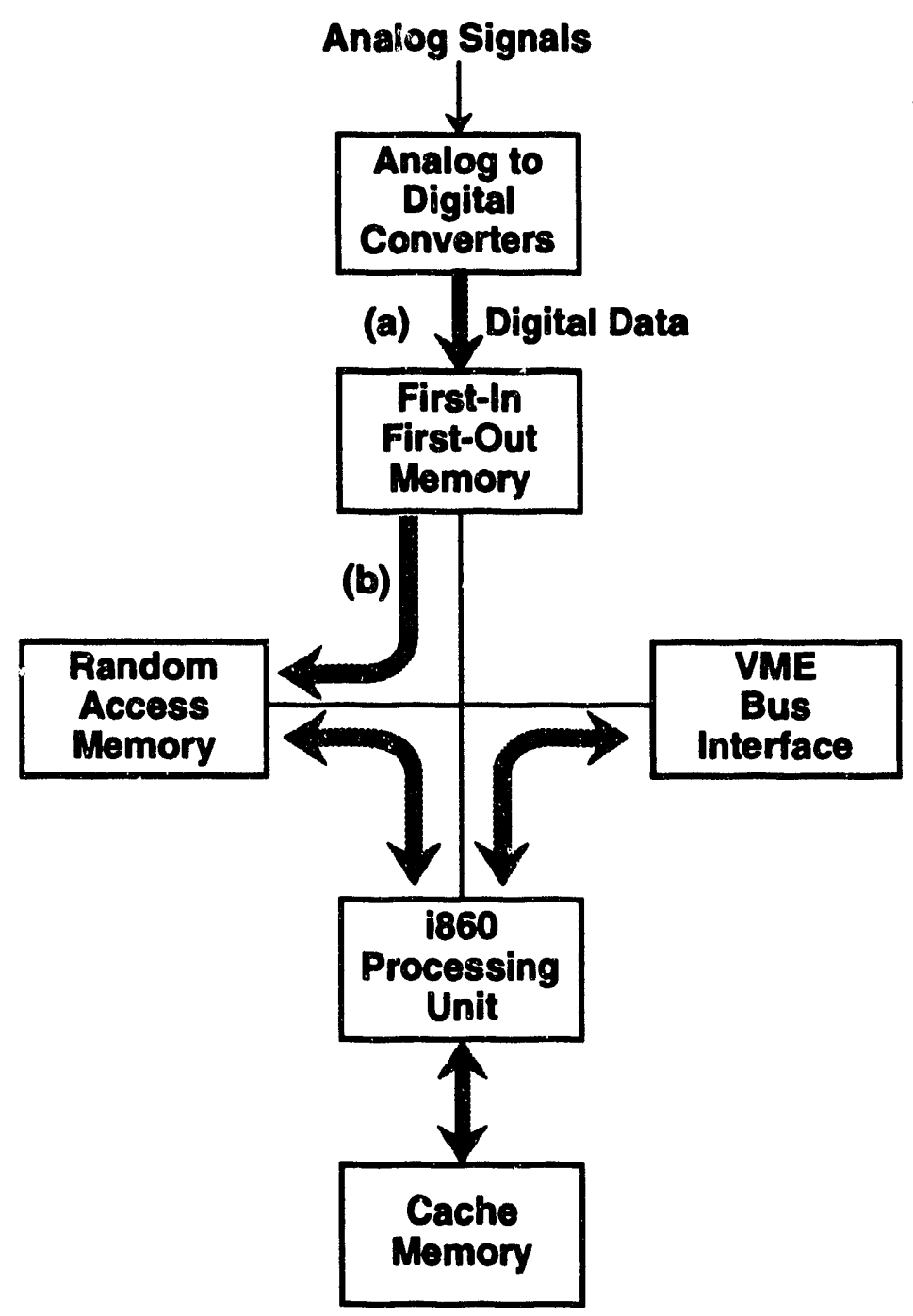

Fig. 4. A simplified diagram of the data flow paths (double width lines) in the data acquisition and processing hardware, and the common connection between the FIFO memory, the random access memory, the i860 processor, and the VME bus interface. There is a separate data path between the i860 processor and the cache memory; data transfer is possible on this path at any time. The data rate on path (a) depends on the application; data are transferred in bursts at the maximum rate of $40 \mathrm{Msamples} / \mathrm{sec}$ in a typical feedback control application but at a lower, continuous, average rate in a typical data reduction application. Data are always transferred at the maximum rate on path (b) in order to minimize the use of the common connection. 
many $8 \mu \mathrm{sec}$ transfer periods) is approximately 36 to 38 million samples per second. This is an estimated value that has not been verified by tests.

Stop mode 3 (see Subsection 3.2) is provided to allow transfer of a specified number of data values with the access of the $\mathrm{i} 600$ processor to memory blocked for the least amount of time. Because the rate of transfer of data into the FIFO memory will, in general, be less than the rate of transfer from the FIFO memory into the SuperCard-2 memory, a DMA transfer operation that continues for a sufficient length of time will eyentually empty the FIFO memory. In stop mode 3, the daughter board will give up control of the SuperCard-2 memory when the FIFO memory becomes empty so that the i860 processor can then access the memory while more data are transferred into the FIFO memory. Typically, then, start mode 2 will be used so that the DMA transfer will resume when the FIFO memory becomes more than half full so that there is a substantial amount of data available to transfer to the SuperCard-2 memory in an efficient, long, burst.

\subsection{The i860 Cache and Data Acquisition}

The i860 processor uses a data cache from which data are fetched if present there. Because the FIFO memory board does DMA cycles which are not detected automatically by the i860 processor, the content of a SuperCard-2 memory location could change so that the copy of that location which is held in cache is incorrect. Data acquisition programs must be written to avoid this problem.

The programmer must ensure that memory locations in the buffer in SuperCard-2 memory into which the data are written by the daughter board board DMA operation are never held in the data cache. There are two ways to do this.

- The SuperCard-2 host software has a provision to declare that the i860 cache should never be used. This is not a good solution when speed is requirea but it allows SuperCard-2 programs to be written in a high level language.

- Always read the data buffer using the i860 pfld instruction. Data read with this instruction are never placed in the data cache. This requires that the portion of code which accesses the data buffer be written in assembly language to ensure that this instruction is used.

Note also, that there must be a space of 32 bytes between the data buffer and the nearest storage area for a variable which could be read into cache. The 32 byte space, which must exist both preceding and following the data buffer, provides one "cache line" of space around the data buffer to ensure that part of the buffer is not read into memory as part of a cache line read.

The daughter board registers and the SuperCard-2 memory are treated differently with respect to the cache memory. The daughter board register content is never placed in cache memory so that when the daughter board registers are read, the correct value is always obtained without taking any special steps. 


\subsection{Accessing the Clock Counter}

Normally the clock counter is used to record the time of the last data acquisition cycle so that the processor knows when the currant data set was acquired. The current value of the clock counter can be obtained at any time if the processor simply needs to know the current time.

To read the clock counter, the counter value must first be latched into the TR register and then it can be read from the TR register. To request that the clock counter value be latched, write a 1 to the CSRTRLATCH bit (see Subsection 3.3). This sets a "request" to latch the clock counter. The clock counter is only latched after a transition of the clock signal from 1 to zero. This means that the clock counter value can only be obtained if there is an actively oscillating clock signal.

After the request to latch is made, the CSRTRLATCH bit should be polled to detect when it is returned to 0 . This indicates that the clock counter value has been latched into the TR register.

The TR register is read with two successive reads. First, do a read to the address of the TR register (see Subsection 3.1). This read returns nonsense data. Then, immediately do a second read, either to the TR register address or another address in control memory which is unused (see Subsection 3.4 for a suggested value). The second read contains the valid TR register value. The programmer should ensure that a DMA write operation cannot occur between the read at the TR register address and the second read at the dummy address by, for instance, disabling DMA operations.

Note that the TR register can be read at any time. However, it is only immediately following a clock counter latch that the $\mathrm{TR}$ register contains timely data.

\subsection{Detecting the End of a DMA Write Operation}

A DMA write operation is normally triggered asynchronously, and as a result the $i 860$ processor has no way of detecting when the DMA write operation starts and how long the DMA write will take. Thus the application program must set up the proper conditions for the DMA write operation to be triggered and then simply wait until the end of the DMA write operation is detected.

There are fours ways to detect the end of the DMA write.

1. The i860 processor can poll the CSRNEWDAT bit in the CSR2 register (see Subsection 3.3). This bit is set at the end of a DMA write operation (except when stop mode 3 is used, for which the behavior is slightly different). This bit can only be cleared through a write of 0 by the the $i 860$ processor.

2. If the value in the TR register is to be written in the data buffer after the data from the FIFO memory, the 1860 processor can set the location that will contain the TR register value to 0 and then poll that location for a change in its value. 
3 In any given 16 bit memory location that will be written with data from the FIFO memory, one or both of the upper two bits can be set to 1 and monitored for a change to 0 . Since the input data values are only 14 bits wide, these two bits will never contain useful data and are always written with 0 during the DMA write operation. This method is not reliable, however, in certain modes of daughter board operation (such as stop mode 3, for instance) because there could be a pause in the DMA write operation between a write to a given memory location and the actual end of the DMA write operation.

4. Whenever the CSRNEWDAT bit is set, the daughter board requests an interrupt of the i860 processor. If the appropriate bit in the SuperCard-2 CSR register is set properly, the interrupt will be enabled. The provision for interrupt driven detection of the end of a DMA write operation is thus present in the hardware, but the sofiware does not exist at this time to handle the interrupt.

\subsection{Behavior When There Is No Digitai Data Source}

The FIFO memory daughter board can be accessed when there are no cable connections to an external digital data source. In this situation, however, the external control signals will be held in the active high state. Thus it will appear that the start trigger is always active and the counter latch will always be active. If start mode 1 is enabled, a DMA transfer will always start because the start trigger will appear to be active. Stop mode 0 will not work because the start trigger will not go inactive. Because there will be no running counter clock signal, the CSRTRLATCH bit will always be set.

\subsection{SuperCard-2 Memory Page Boundaries}

The SuperCard-2 memory is organized into "pages," blocks of memory 4096 bytes in length. Access to the SuperCard-2 memory can proceed at the maximum rate only as long as sequential memory accesses are to locations in the same memory page. When the memory address jumps from one page to another there is a small delay of about $175 \mathrm{nsec}$. In addition, during this delay a memory refresh can occur, lengthening the delay.

When choosing the location for a DMA transfer buffer, then, it is optimum to choose the memory buffer start address and length so that the SuperCard-2 memory page does not change during the DMA transfer. Of course, this is not possible if the amount of data to be transferred is large. Note that memory pages begin at byte addresses divisible by 4096 , but the value loaded into the daughter board address counter to indicate the start of a memory page is divisible by 512 because the address counter indicates groups of 8 bytes. 


\section{Programming for the FIFO Memory Daughter Board}

This section deicribes the programmer's view of the FIFO memory ciaughter board. The various registers on the FIFO memory board are described. The programs that access the acquisition system hardware run on the $i 860$ processor.

\subsection{Addressing the Data Acquisition System}

The FIFO memory daughter board as viewed from the i8t 0 processor consists of four registers which are accessed in the "control memory" address region of the SuperCard-2. These registers and the values of their absolute addresses in SuperCard-2 address space are listed in Table 2. Briefly, the registers are the following:

- The control register (CSR). This 24 bit read/write register contains bits which control the function of the daughter board.

- The second control and status register (CSR2). This 32 bit read/write register contains bits which control the function of the daughter board system and indicate status. Part of this register is the data transfer counter (or "sample counter").

- The clock counter latched value register (TR). This 32 bit read only register contains the value that was in the 32 bit clock counter the last time the value was latched. The value is latched either by an external signal (the "clock latch" signal) or by a request generated by setting a CSR2 bit.

- The data buffer address register (AD). Tris 21 bit write only register contains the byte address (divided by 8 ) that will be loaded into the address counter in order to specify the buffer in the SuperCard-2 memory into which the FIFO memory data will be written. Note that the value in this register is actually an offset giving the number of blocks of 8 bytes between address 0 and the start of the data buffer.

Table 2

The FIFO Memory Daughter Board Registers

\begin{tabular}{lccc}
\hline \multicolumn{1}{c}{ Register Name } & Abbreviation & Address (hex) & Read and/or Write \\
\hline Control & CSR & fbr80020 & read/write \\
Control and status & CSR2 & fbf8003c & read/write \\
Clock counter latched value & TR & fbf80028 & read only \\
Data buffer address & AD & fbf80034 & write only \\
\hline
\end{tabular}




\subsection{The Control Register}

The values of the bits in the CSR register control the operation of the data acquisition system. This register must always be accessed with a 32 bit (one word) operation. Of the 32 bits returned from a register read, only the least significant 24 bits contain useful data. Table 3 summarizes the meaning of each of the bits in this register. (Note that in this table, bit 0 is the least significant).

Table 3

The Bit Fields in the CSR

\begin{tabular}{|c|c|c|c|}
\hline $\begin{array}{c}\text { Bit } \\
\text { Numbers }\end{array}$ & $\begin{array}{l}\text { Bit Field } \\
\text { Name }\end{array}$ & Function & $\mathbf{R} / \mathbf{W}$ \\
\hline 0 to 7 & ID & Daughter board identification number & read only \\
\hline 8 to 11 & $\mathbf{P}$ & The "write input bus" mask & $\mathbf{R} / \mathbf{W}$ \\
\hline 12 to 16 & PCNTRL & Initial value of $P$ mask counter & $\mathbf{R} / \mathbf{W}$ \\
\hline 17 & CSRCNTRINE & $0 / 1=$ disable/enable write of TR with data & $\mathbf{R} / \mathbf{W}$ \\
\hline 18 & CSRUA & $0 / 1=$ disable/enable address counter update & $\mathrm{R} / \mathrm{W}$ \\
\hline 19 & CSRPT & $0 / 1=$ disable/enable pass through mode & $\mathbf{R} / \mathbf{W}$ \\
\hline 20 to 21 & CSRSTART & Specify the trigger for the start of a write cycle & $\mathbf{R} / \mathbf{W}$ \\
\hline 22 to 23 & CSRSTOP & Specify the trigger to stop a write cycle & $\mathbf{R} / \mathbf{W}$ \\
\hline
\end{tabular}

The CSR can be read and written. Bits 8 through 23 always return the last value written there. Bits 0 through 7 are actually read only. When written, these bits are not altered. When the CSR register is read, bits 24 through 31 are undefined.

During a write of the CSR, all bits are altered at once. Therefore, if it is necessary to change only specific bits of the CSR, the CSR should be read first and the required bits changed, and then the new value written to the CSR.

A detailed description of each of the CSR bit fields is as follows:

ID The ID field contains the daughter board identification code. This value is meant to indicate to software the type of daughter board which is installed. For the FIFO memory board this field will always be of (hex). If the field reads 0 or $\mathrm{fr}$ then the daughter board is either not installed or is not functioning properly. This field is never altered when the CSR is written so its value during a CSR write is arbitrary. 
$\mathrm{P}$ The $\mathrm{P}$ field is a bit mask that indicates the digitizer buses which should be written to the FIFO memory. Figure 1 shows that the input data buses for the daughter board are connected in two sets of four. The $P$ mask indicates whether the data on a given bus in each of the sets of four should actually be written to the FIFO memory. If there are no data to be provided on a given bus, then data from this bus should not be written to the FIFO memory because this will produce a value of nonsense data in the memory data buffer. If a bit in the $\mathrm{P}$ mask is zero, then data from that bus in each of the two sets of four buses is not written to the FIFO memory. A one in the mask enables the data to be written to the FIFO memory. The user's manual for each digital data source should be consulted to determine what data source is connected to each bus. The $P$ field is ignored unless the "P counter" has a value of 0 .

PCNTRL The PCNTRL (P counter load value) field gives the initial value to be loacied into a five-bit counter that indicates when the $P$ mask should actually be used to enable or disable a data write to the FIFO memory. The counter is initialized with the PCNTRL field value when the start trigger input signal line from the external data source is inactive. After the start trigger becomes active, the counter is decremented once each time data are written to the FIFO memory (i.e., on each cycle of the data accept trigger). After the counter value reaches 0 , the counter stops decrementing and the $P$ mask field is used for all following cycles of the data accept trigger until the start trigger becomes inactive again.

CSRCNTRWE When this bit is 1, at the end of a DMA write of data to the SuperCard-2 memory, the value in the TR register is written to the data buffer in memory in the location following the data from the FIFO memory. If the bit is 0 , then the TR register value is not written to SuperCard-2 memory.

CSRU A When this bit is 1 , the value in $\mathrm{AD}$ register is written to the address counter at the start of each DMA write cycle. If this bit is 0 , then the address counter is not altered and the address to which data are written continues to increment with each DMA cycle.

CSRPT When this bit is 1 , the daughter board operates in pass through mode. In this mode, DMA operations operate normally except that the data are not actually written to the SuperCard-2 memory and access of the i860 processor to the memory is not blocked during a "DMA write" operation (see Subsection 3.7).

CSRSTART This bit field specifies a two-bit mode number which determines the method by which a DMA write cycle can be triggered. The four modes are as follows.

mode 0: CSRSTART is binary 00. In this mode DMA write cycles are disabled.

mode 1: CSRSTART is binary 01. In this mode a DMA write cycle is triggered by a high level on the start trigger input signal from the external data source.

mode 2: CSRSTART is binary 10. In this mode a DMA write cycle is begun when the FIFO memory is more than half full. 
mode 3: CSRSTART is binary 11. In this mode a DMA write cycle is triggered by the i860 processor, that is, a write cycle begins immediately after the 11 bit pattern is written to the CSRSTART bits in the CSR. A write cycle will start automatically as long as this mode is set unless the CSRHOLDENA bit (see CSR2 description in Subsection 3.3) is set. In that case a new DMA write cycle cannot begin as long as the CSRNEWDAT bit is set, allowing the i860 to alter the start mode bits between DMA write cycles.

CSRSTOP This bit field specifies a two bit mode number which determines the method or methods by which a DMA write cycle is terminated. Independent of the stop mode which is programmed, it is always the case that the DMA write cycle will be terminated when the sample counter (see the description of the SC bit field in CSR2 in Subsection 3.3) reaches its terminal count value. The four modes are as follows.

mode 0: CSRSTOP is binary 00. In this mode a write cycle is terminated when the start trigger signal from the external data source is inactive and the FIFO memory is empty. The CSRNEWDAT bit in CSR2 is set at the end of the write cycle.

mode 1: CSRSTOP is binary 01. In this mode the write cycle stops when the FIFO memory becomes empty. The CSRNEWDAT bit in CSR2 is set at the end of the write cycle.

mode 2: CSRSTOP is binary 10. In this mode the write cycle ends when the sample counter reaches its terminal count value. The CSRNEWDAT bit in CSR2 is set at the end of the write cycle.

mode 3: CSRSTOP is binary 11. In this mode a DMA write cycle is terminated either when the FIFO memory is empty or when the sample counter in CSR2 reaches its terminal count value. However, the CSRNEWDAT bit in CSR2 is set and the TR register is written to SuperCard-2 memory (if CSRCNTRWE is set) only when a write cycle ends because the sample counter reaches terminal count. This mode is intended for an application where an amount of data specified by the sample counter is to be collected, but the DMA write to SuperCard-2 memory can pause when there is no data in the FIFO memory. The $i 860$ then has access to the SuperCard-2 memory during this period. The DMA transfer is not considered complete, however, until the amount of data specified by the value in the sample counter has been collected. CSRNEWDAT is set at that time.

\subsection{The Control and Status Register Number 2}

This register has some bits which specify how the daughter board should operate and some bits which reflect the status of the daughter board. This register must always be accessed with a 32 bit (one word) operation. When read, all 32 bits return significant information. Table 4 summarizes the meaning of each of the bits in this register. 
Table 4

The Bit Fields in the CSR2 Register

\begin{tabular}{|c|c|c|c|}
\hline $\begin{array}{c}\text { Bit } \\
\text { Numbers }\end{array}$ & $\begin{array}{l}\text { Bit Field } \\
\text { Name }\end{array}$ & Function & $\mathbf{R} / \mathbf{W}$ \\
\hline 0 & CSRRESET & $1=$ hold the daughter board in reset state & $\mathbf{R} / \mathbf{W}$ \\
\hline 1 & CSRNEWDAT & Set at completion of a DMA write cycle & $\mathbf{R} / \mathbf{W}$ \\
\hline 2 & CSRSAMPDC & $0 / 1=A$ trigger for the data source & $\mathbf{R} / \mathbf{W}$ \\
\hline 3 & CSREXTRESET & $1=$ hold the external data source reset & $\mathbf{R} / \mathbf{W}$ \\
\hline 4 & CSRTRLATCH & $\begin{array}{l}1=\text { request that the clock counter value } \\
\text { be latched }\end{array}$ & $\mathbf{R} / \mathbf{W}$ \\
\hline 5 & CSRFIFOFULL & Set if the FIFO memory becomes full & $\mathbf{R} / \mathbf{W}$ \\
\hline 6 & CSRCM & $1=$ use continuous memory mode & $\mathbf{R} / \mathbf{W}$ \\
\hline 7 & CSRHOLDENA & $\begin{array}{l}\text { 1=a new write cycle cannot start when } \\
\text { CSRNEWDAT is set }\end{array}$ & $\mathbf{R} / \mathbf{W}$ \\
\hline 8 to 31 & sc & The 24 bit sample counter & $\mathbf{R} / \mathbf{W}$ \\
\hline
\end{tabular}

The CSR2 can be read and written (except that writes to this register are ignored when the CSRPT bit is set, see Subsection 3.7). During a write to the CSR2 register, all bits are altered at once. Therefore if it is necessary to change only specific bits of the CSR2, the CSR2 register should be read first, the required bits changed, and then the new value written to the CSR2. However, two of the bits cannot be set by a write operation and one cannot be cleared by a write operation. These are bits (specified below) which can be altered asynchronously by the daughter board and, when this is possible, should not be altered by the i860 processor.

A detailed description of each of the CSR2 bit fields is as follows.

CSRRESET When this bit is 1, the FIFO memory board is held reset. In this case, the only function that can be performed by the FIFO memory board is an access to the CSR, CSR2, TR, and AD registers. A reset of the FIFO memory board before normal operation is recommended. The CSRRESET bit is set at power-up and whenever the SuperCard-2 is reset by the host processor, or the bit can be set by the i860. The bit is cleared only by a write to the CSR2 by the 1860 processor. Some CSR2 bits are held cleared or set and the FIFO memory is emptied when CSRRESET is set.

CSRNEWDAT This bit is set at the end of a DMA write cycle to indicate that new data are available in the SuperCard-2 memory data buffer. This bit is reset either by writing a 0 into it or by a reset of the FIFO memory board. See the description of the CSRSTOP bits in the CSR register in Subsection 3.2 for details about when this bit is set. If this bit is set and CSRHOLDENA is set then a new DMA write cycle cannot be started. 
Whenever CSRNEWDAT is set, a request is made to interrupt the $\mathrm{i} 860$. Thus, if the appropriate bit in the SuperCard-2 interrupt enable register is set, the i860 processor can be interrupted at the end of a DMA write cycle.

It is only possible to write a 0 to this bit. If an attempt is made to write a 1 to this bit, the bit value is not altered. Thus, if it is desired to alter other bits in CSR2 without changing the state of CSRNEWDAT then, when the other bits are written, the data for this bit must be 1 . This is necessary because the daughter board can asynchronously set this bit.

This bit is held clear when CSRRESET is set.

CSRSAMPDC This bit is intended to be a trigger for the digital data source. A transition of CSRSAMPDC from 0 to 1 provides a trigger signal. This bit is always cleared automatically when CSRNEWDAT is set, or the bit can be cleared by the i 860 processor. If several FIFO memory daughter boards are daisy chained together on the same input cable set, any of the daughter boards can drive the signal line to the external data source by setting this bit.

This bit is held clear during a reset of the daughter board, i.e., when CSRRESET is set. If CSRRESET is set, it is not possible to clear CSRRESET and set CSRSAMPDC with a single write to the CSR2 register. It is necessary to first write a 0 to CSRRESET and then, in a separate write to CSR2, put a 1 in CSRSAMPDC.

CSREXTRESET This bit is intended to be a reset control for the external data source. When this bit is set the external data source is held reset (see the manual for the external data source for details). This bit can be both set and cleared by the 1860 processor. If several FIFO memory daughter boards are daisy chained together on the same input cable set, any of the daughter boards can drive the signal line to the external data source by setting this bit.

This bit is cleared at power up and held clear when the SuperCard-2 holds the daughter board reset (typically when no program is running on the SuperCard-2). This bit is not affected by CSRRESET. This scheme prevents an inactive SuperCard-2/FIFO memory board pair from holding the data source reset when multiple FIFO memory daughter boards are daisy-chained.

CSRTRLATCH When this bit is 1 , the clock counter content will be latched after the next falling edge of the input clock. This bit is set to a 1 either by the external "clock latch" signal or by writing a 1 during a CSR2 write cycle. The bit is set to 0 by the FIFO memory board hardware after the clock counter value has been latched. 
It is only possible to write a 1 to this bit. If an attempt is made to write a 0 to this bit, the bit value is not altered. Thus, if it is desired to alter other bits in CSR2 without changing the state of CSRTRLATCH then, when the other bits are written, the data for this bit must be 0 . This is necessary because the daughter board can asynchronously clear this bit.

This bit is held clear during a reset of the daughter board, i.e., when CSRRESET is set. If CSRRESET is set, it is not possible to clear CSRRESET and set CSRTRLATCH with a single write to the CSR2 register. It is necessary to first write a 0 to CSRRESET and then, in a separate write to CSR2, put a 1 in CSRTRLATCH.

CSRFIFOFULL This bit is set to 1 whenever the FIFO memory becomes full. When the FIFO memory is full writes into the memory are ignored, so if CSRFIFOFULL is detected to be a 1 there is a chance that some data were lost. This bit is reset to 0 either by a write by the 1860 processor or by a daughter board reset.

It is only possible to write a 0 to this bit. If an attempt is made to write a 1 to this bit, the bit value is not altered. Thus, if it is desired to alter other bits in CSR2 without changing the state of CSRFIFOFULL then, when the other bits are written, the data for this bit must be 1 . This is necessary because the daughter board can asynchronously set this bit.

This bit is held clear during a reset of the daughter board, i.e., when CSRRESET is set.

CSRCM When this bit is 1 , "continuous memory" mode is enabled. In this mode data are writcen to every location in the SuperCard-2 memory rather than into every other pair of bytes as is normally done to accommodate the integer to floating point conversion algorithm described in Subsection 2.4. See Subsection 3.6 for a detailed description of continuous memory mode.

CSRHOLDENA When this bit is 1, a new DMA write cycle cannot start when CSRNEWDAT is set. This bit is used to ensure that a new DMA write cannot follow a previous DMA write cycle until the 1860 processor has had a chance to be alerted that new data are available from the previous write cycle. CSRHOLDENA can thus be used to prevent new data in a DMA buffer from being overwritten before the i860 processor has access to it.

SC The sample counter (or SC, also referred to in this document as the data transfer counter) is used to record how many data values have been written to the SuperCard-2 memory. For each write to SuperCard-2 memory the SC is incremented. Note that each increment indicates that two data samples have been written since two samples are written in parallel during each write to SuperCard-2 memory. The SC contains 24 bits so it is possible to count to 16 million, representing more data than the SuperCard-2 memory can hold. Therefore, if the SC is set to 0 before the start of a DMA write cycle, it simply records the amount of data written to SuperCard-2 memory. The i860 processor 
can read this register and use this information in its data processing. For instance, depending on the application, when stop mode 1 is used, it may not be possible to know ahead of time how much data will be written in a DMA write cycle. The sample counter can be read after the DMA write cycle completes to determine the exact count of data values written.

The maximum value that the SC can hold is $2^{24}-1$. When the counter is incremented once past this vaiue so tirat the total number of counts recorded is $2^{24}$ (but the counter value actually overflows to be 0 ) the current DMA write cycle is terminated. When the initiai value of the SC is zitro it is not possible for the SC to reach the maximum value before the SuperCard-2 memory is full so in this case, in a properly functioning data acquisition algorithm, the maximum count value of the SC will never stop a DMA write cycle. To use the SC to stop a write cycle, initialize the SC with $2^{24}$ minus the number of pairs of data values that should be written to SuperCard-2 memory in the next DMA write cycle.

\subsection{The Clock Counter Value Registar}

The value of the clocis signal cointer can be latched at any time. The latch occurs whenever the external clock latch signal is activated or after a 1 is written to the CSRTRLATCH bit. The latched value is placed into the clocis counter value register (TR). This value can be read at any time. Note that the clock counter value is latched only following the falling edge of the input clock signal. Thus, in order to latch the clock counter an operating input clock is required. If the i860 processor sets the CSRTRLATCH bit in order to trigger a clock counter value latch, the CSRTRLATCH bit should be polled to detect when it is reset to zero, indicating that the latch operation is complete.

To obtain the value in the TR register, two reads are necessary. The first read must be addressed to the TR register. The address in the second read can be to any unused address in the SuperCard-2 control memory address space (e.g., 0xfbf80040). The result of the first read is undefined. The second read contains the correct TR value. All 32 bits are significant.

Writes to the TR registar are ignored.

\subsection{The Data Buffer Address Register}

The data buffer address register (AD) contains the value that will be loaded into the address counter at the beginning of a DMA cycle (if CSRUA is set). The value indicates the first location in the SuperCard-2 memory where data will be placed during a DMA write cycle. The value in the $A D$ must be a valid meinory address divided by eight. That is, the $A D$ contains the number of blocks of 8 bytes between address 0 and the start of the buffer where data are to be written. This arrangement exists because the data bus between the daughter board and the SuperCard is 8 bytes wide. 
At the start of a DMA write cycle, the $A D$ value is optionally copiecl to the address counter which is then used to provide the current memory address as data are written to the SuperCard-2 memory. After each write to the SuperCard-2 memory, the address counter is incremented (unless continuous memory mode is in use, see Subsection 3.6). At the end of the DMA write cycle, the counter contains the address of the first memory location following the buffer containing the data that were just written. If the $A D$ value is copied to the counter at the beginning of each DMA cycle, then the same memory buffer is used for each DMA cycle until the $A D$ value is changed. If the $A D$ value is not copied to the counter, then the data buffer used on a given DMA cycle is located immediately after the buffer used in the previous DMA write cycle. This provides two modes of operation: (1) a conventional digitizer mode where the data are written to a different location on each cycle so that all data are saved and (2) a mode where the data are written to a fixed buffer each time assuming that the data are processed and discarded between DMA write cycles.

Only the lower 21 bits of the $A D$ are used, the upper bits are ignored. However, the $A D$ must always be accessed with a 32 bit write. Reads of the $A D$ are ignored. When the $A D$ register is written with a new value, the same value is simultaneously loaded into the address counter.

Writes to this register are ignored when the CSRPI bit is set.

\subsection{Continuous Memory Mode}

There are two possible ways in which data are written into the SuperCard-2 memory.

1. In the default mode (the CSRCM bit in CSR2 is 0 ), two 16 bit data values are written to SuperCard-2 memory at a time. These values are written into the memory which maps into bytes $0,1,4$, and 5 of the 64 bit wide interface between the daughter board and the SuperCard-2. This is illustrated in Fig. 3. The memory which maps into bytes $2,3,6$, and 7 is not altered during a write cycle. The address counter on the daughter board is incremented after each write to SuperCard-2 memory.

This method of transferring data to SuperCard-2 memory facilitates the algorithm for conversion from integer to floating point format. This method is appropriate for use when the data written to SuperCard-2 memory is to be processed immediately by the i860 processor. However, if the object is to simply transfer data to the SuperCard-2 memory and store it there, this method is not optimum because data are actually written to only half of the memory.

2. In continuous memory mode, every byte of memory on the SuperCard-2 can be filled with data. To choose this mode, set the CSRCM bit in the CSR2 to 1. As in the default mode, two 16 bit data values are written to the SuperCard-2 memory at a time. However, the address counter increments after only every other write so that it points to the same group of 8 bytes for two consecutive writes to the SuperCard-2 memory. On the first write, data are placed in the memory which maps to bytes $2,3,6$, and 7 of the 
64 bit daughter board interface and bytes $0,1,4$, and 5 are not altered. On the second write, data are placed in bytes $0,1,4$, and 5 and bytes $2,3,6$, and 7 are not altered. Again, the byte numbers refer to the illustration in Fig. 3.

The continuous memory mode accommodates applications where the SuperCard-2 memory is used to buffer a large amount of data for "near real time" processing in which more time is available for the subsequent conversion to floating point format and data processing. In this case all of the memory can be used to hold data.

Note that in this mode of operation, it is possible to have an odd number of data writes during a DMA write cycle. In this case, assuming that the first data were written to bytes $2,3,6$, and 7 , there are no data in bytes $0,1,4$, and 5 at the address indicated by the address counter at the end of the DMA write cycle. If the CSRUA bit is not set, then these unused bytes will be the first bytes used during the next DMA. write cycle (unless CSRCNTRWE is set so that the TR register content is written also, see below). If the CSRUA bit is set, then the next DMA write cycle will start over at the beginning of the buffer indicated by the address in the $A D$ register.

It is important to note that the flag which indicates which half of the data bus to use is initialized properly for continuous memory mode only when the address counter is loaded; that is, when the $\mathrm{AD}$ register is loaded or at the beginning of a DMA write cycle with CSRUA set. Thus, if continuous memory mode is to be used, CSRCM must be set before the address counter is loaded. When the address counter is loaded and CSRCM is set, the flag always initializes to point to bytes $2,3,6$, and 7 so that data is written to those bytes first. When CSRCM is cleared, the flag is immediately reset to point to bytes $0,1,4$, and 5 (i.e., there is no wait for the address counter to be loaded).

There is special behavior when the value in the TR register is to be written to memory at the end of a DMA write cycle (CSRCNTRWE is set). If there is an odd number of writes during the DMA write cycle so that there is an unused set of bytes, this set of bytes is skipped because the TR value requires 32 bits of storage. If there is an even number of bytes written during the DMA write cycle, the TR value is simply written to the 32 bits (bytes 4, 5, 6, and 7) which follow the last bytes written with data. In continuous memory mode, after the TR value is written, the address counter is always left pointing to bytes $2,3,6,7$, of the 8 byte set following the set where the TR register value was written.

\subsection{Pass Through Mode}

The CSR bit CSRPT, when set, enables pass through mode. When pass through mode is enabled, the daughter board behaves in the normal way when performing a "DMA write" operation except that no data are actually written to the SuperCard-2 memory. Access to the SuperCard-2 memory is not requested by the daughter board so that access of the i860 processor to the SuperCard-2 memory is not restricted during a DMA write operation in pass through mode. 
Pass through mode is included for situations when it is desired to ignore a well known amount of input data. An example of a situation where this is useful is a data reduction application that is executing on two SuperCard-2 boards in parallel, each with its own FIFO memory daughter board. Each of the $i 860$ processors operates on alternating sets of input data. A given processor acquires a set of data and then analyzes it while the next set of data arrives and is ignored. The other $i 860$ processor acquires the set of data that the first processor ignored and analyzes it. The set of data that is ignored can be acquired in the same way as the set that is analyzed, except that it is acquired in pass through mode so that it is not written to the SuperCard-2 memory and the access of the i860 processor to memory is not blocked during the data acquisition.

The i860 processor can access the daughter board registers during a pass through mode operation because the daughter board port on the SuperCard-2 is not actually busy with the "DMA write" operation. However, to avoid disturbing the DMA operation in progress, certain operations are ignored when the daughter board is in pass through mode.

- Reads and writes of the CSR register are performed normally.

- Reads of the CSR2 register are performed normally but writes of the CSR2 register are ignored.

- Writes to the $A D$ register are ignored (reads of the $A D$ are always ignored).

- Reads of the TR register are performed normally but because the CSR2 register cannot be written, a latch of the clock counter value cannot be triggered by the $i 860$ processor. Only the external trigger signal can cause the TR value to change while the daughter board is in pass through mode.

Note that the daughter board enters pass through mode immediately after the CSRPT bit is set in the CSR register. Therefore, the first operation in setting up a DMA write operation in pass through mode should be to write the CSR register with 0 in the CSRSTART bits to disable the start of a DMA write cycle and 0 in CSRPT. Then write the proper values to the CSR2 and AD registers. Finally, write the CSR register with CSRPT set and the proper start mode chosen.

\subsection{Ordering of Data in the SuperCard-2 Input Memory Buffer}

As described in Section 2, the digital input data are obtained on eight parallel buses. The data are written into a buffer in the SuperCard-2 memory at the location given by the address counter. This section describes the order in which the data appear in this memory buffer. 
As shown in Fig. 1, the eight input data buses for the FIFO memory daughter board are divided into two groups of four. The groups are referred to by number, 1 or 2 , and within each group the buses are labeled A, B, C, or D (it is necessary to consult the manual for the particular data source to determine how the eight data buses are connected to the digital data source).

The order in which the data appear in the buffer, from the point of view of the programmer, is illustrated in Fig. 5(a) through (c).

For the situation when continuous memory mode is not in use, refer to Fig. 5(a) and (b). If the memory buffer is address $d$ as a contiguous array of 32 bit words, Fig. 5 (a) applies, and the data from the input buses appear in the memory buffer in the following order: $2 \mathrm{~A}$, $1 \mathrm{~A}, 2 \mathrm{~B}, 1 \mathrm{~B}, 2 \mathrm{C}, 1 \mathrm{C}, 2 \mathrm{D}, 1 \mathrm{D}$. Note that only every other pair of bytes in the memory buffer is actually altered during the DMA write by the daughter board (see Subsection 2.4). Thus, if the memory buffer is viewed as a contiguous array of 16 bit integers, the illustration in Fig. 5(b) applies. In this case the first integer in the buffer is not altered, then the data from bus $2 \mathrm{~A}$ appears, followed by another unaltered integer, then the data from bus $1 \mathrm{~A}$, etc.

If continuous memory mode is in use, the data from each of the bus groups is compressed to fill the memory; all bytes in the buffer are altered during the DMA write by the daughter board. In this case, the buffer should be treated as a contiguous array of 16 bit integers, and the order of data in the buffer is illustrated in Fig. 5(c). The data from the input buses appear in the memory buffer in the following order: $2 \mathrm{~A}, 2 \mathrm{~B}, 1 \mathrm{~A}, 1 \mathrm{~B}, 2 \mathrm{C}, 2 \mathrm{D}, 1 \mathrm{C}, 1 \mathrm{D}$.

A Fortran array can easily describe the structure of the data buffer. In the normal data write mode (not continuous memory mode), the buffer can be dimensioned

\section{INTEGER*4 DATA $(2,4, N)$}

where $\mathrm{N}$ indicates the number of sets of eight data values present in the data buffer. To access the array use DATA $(I, J, K)$, where $I$ is the group index, and $J$ is the set label ( 1 through 4 to indicate $A$ through $D$ ). Here, to get the data from group 2, I is 1 , and to get the data from group $1, I$ is 2 . Note that there is only one data sample in each group of four bytes.

In continuous memory mode, the array should be

\section{INTEGER*2 DATA $(2,2,2, N)$.}

In this case there are two samples from group 2 in memory followed by two samples from group 1. There is a sample in each group of 2 bytes. To access the array use DATA(L,I,J,K), where $I$ is 1 for group 2 data and $I$ is 2 for group 1 data, $J$ is 1 for sets $A$ and $B$ or 2 for sets $C$ and $D, L$ is an index 1 for sets $A$ and $C$ or 2 for sets $B$ and $D$. 


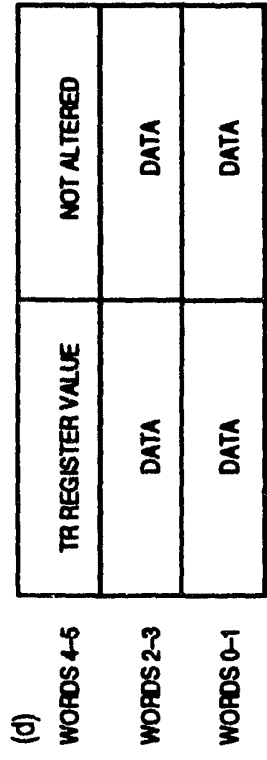

\begin{tabular}{|c|c|c|c|}
\hline $\begin{array}{l}\text { 을 } \\
\text { 3 }\end{array}$ & 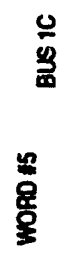 & 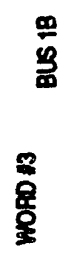 & 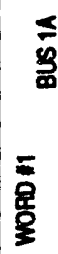 \\
\hline 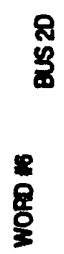 & $\frac{0}{8}$ & $\begin{array}{l}\mathbb{8} \\
3 \\
8 \\
8 \\
8 \\
8\end{array}$ & $\begin{array}{l}\text { న } \\
8 \\
8 \\
8 \\
8 \\
0 \\
0\end{array}$ \\
\hline
\end{tabular}

Ð

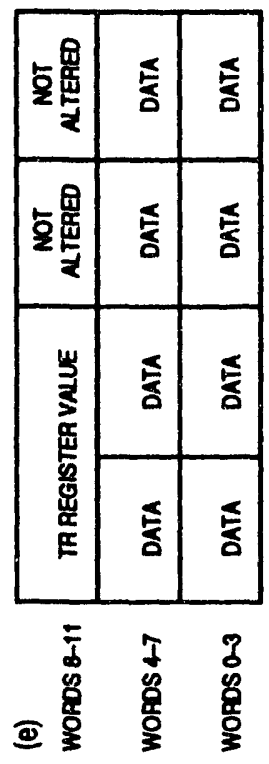

\begin{tabular}{|c|c|c|}
\hline 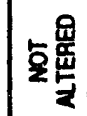 & 点量 & 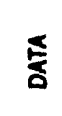 \\
\hline 昼墨 & 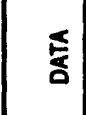 & t \\
\hline 岁 & 送曽 & $\widehat{\mathbf{s}}$ \\
\hline 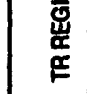 & $\underline{\mathbf{s}}$ & $\widehat{\alpha}$ \\
\hline $\begin{array}{r}\bar{\vdots} \\
\bar{\phi} \\
\overline{0} \\
\equiv\end{array}$ & 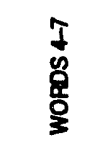 & 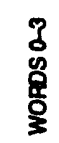 \\
\hline
\end{tabular}

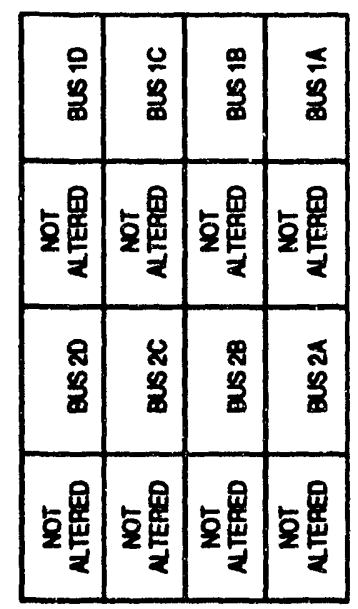

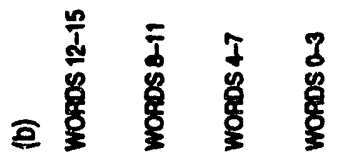

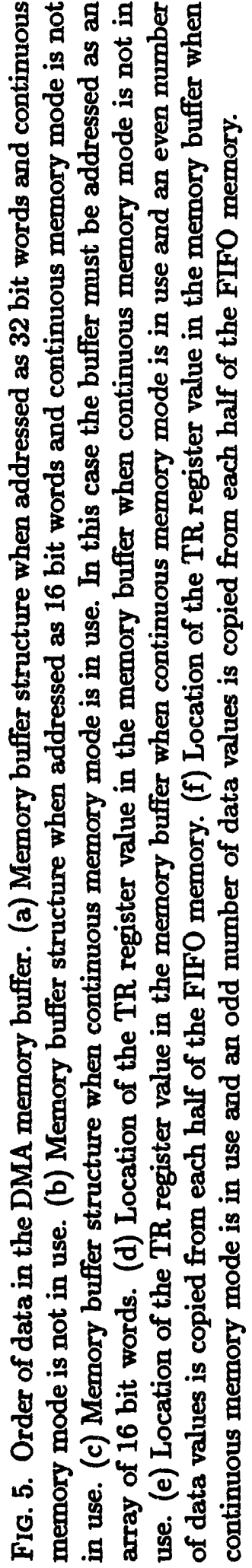


The P and PCNTRL bit fields in the CSR can be used to indicate that the data on one or more input buses should not be written to the FIFO memory (see the discussion of the CSR in Subsection 3.2). In that case, the data not written to the FIFO memory are missing from the data buffer and the order described in the previous paragraph is compressed to fill the space. This provision is made so that unused data buses do not produce gaps in the data buffer.

Figure 5(d) through (f) illustrates the location in the memory buffer of the TR register value when it is written during a DMA write. Figure $5(\mathrm{~d})$ illustrates the case in which continuous memory mode is not in use. The TR register value is placed in the first set of four bytes in the buffer that follow the data copied from the FIFO memory. Figure 5(e) illustrates the case in which continuous memory mode is in use and an even number of data values is copied from each half of the FIFO memory. The TR register value occupies four bytes immediately following the data. Figure 5(f) illustrates in case in which continuous memory mode is in uee and an odd number of data values is copied from each half of the FIFO memory. In this $c$ there are two unused 16 bit integer storage locations that precede the location where the TR register value is stored. In these last two cases the memory buffer is a mixture of 16 bit integers and one 32 bit integer. A C language "structure" or a Fortran language "record" are useful to represent this data structure.

\subsection{Power Up Status}

When the power is initially applied to the VME card cage, the VME "slot 1" circuit generates a reset signal. The SuperCard-2 uses this signal to reset its circuits. The signal is also passed to the daughter board. The reset signal line to the daughter board is also active whenever a specific bit in the SuperCard-2 CSR is set. Whenever the reset signal is active the FIFO memory daughter board CSR2 bit CSRRESET is set so that the FIFO memory board is held reset (see the discussion of CSRRESET in Subsection 3.3 for details on the reset state of the FIFO memory board).

After power-up, the content of the CSR, the SC and the bits in the CSR2 unaffected by a reset are undefined. To ensure that the FIFO memory board remains inactive after power-up, the board is held in the reset state until after the CSRRESET bit is cleared. Therefore, the first writes to the daughter board after power-up should either set the CSR and CSR2 to a desired state, or the first write to CSR2 should explicitly set the CSRRESET bit so that the FIFO memory board continues to be held in the reset state. A harmless way to initialize these registers is to first write 0 to all bits in the CSR and then write zero to all bits in the CSR2. In this way the daughter board is removed from the reset state but all DMA activity is disabled. 


\section{Outlines of Data Acquisition Programs}

In this section, the various steps necessary for data acquisition are described for several possible modes of operation of the daughter board.

\subsection{A Typical Feedback Control Application}

In this type of application the input data should pass through the FIFO memory as quickly as possible. Therefore, start mode 1 is used because in that mode the DMA transfer is typically triggered before the transfer of data to the FIFO memory begins. In this example, stop mode 0 is used but other stop modes might also be appropriate. In stop mode 0 , the data transfer continues until the external data source says that it should stop and does not stop until all data are removed from the FIFO memory. Because the DMA transfer has already started when the first data are placed in the FIFO memory, the data are copied out of the FIFO memory immediately after being written to the FIFO memory. The DMA transfer circuit is always ready to copy the data immediately because the DMA transfer does not stop until it is told to do so by the external data source.

A typical program for data acquisition and analysis would proceed as follows.

1. Write 0 to all bits in the CSR register. This ensures that a DMA write operation cannot be triggered and that pass through mode is not chosen.

2. Reset the data acquisition circuits. Since the software cannot know the exact status of the data acquisition circuits, it is best to reset the system at the start of a data acquisition sequence. This reset will ensure that the FIFO memory is empty and the circuits are cleared of any anomalous condition that might have existed before the start of the program.

To reset the FIFO memory daughter board and the external data source, write $0 \times 9$ to the CSR2 register in order to set the CSRRESET bit and the CSREXTRESET bit (the prefix $0 x$ on a number indicates hexadecimal format). Then write $0 \times 0$ to the CSR2 register to end the reset state.

When the CSRRESET bit is 1 , the values of the other CSR2 bits are not important.

3. Write the $\mathrm{AD}$ register. The content of the $\mathrm{AD}$ register is unknown otherwise because this register cannot be read. Therefore, the address of the DMA buffer should be written to the $\mathrm{AD}$ register at the start of the data acquisition program. This value will not change until the register is written again. 
4. Enable data acquisition. To enable acquisition of data, it is necessary to properly set the CSR and CSR2 bits. To enable functioning of all parts of the circuit, a start mode, and a stop mode must be programmed and CSRRESET must be 0 . Here we will assume that CSRSAMPDC will be used to trigger the external data source so that there is no incoming data until we explicitly set CSRSAMPDC. We will also assume that the CSRHOLDENA bit should be set so that a new DMA transfer cannot follow the previous DMA transfer until the i860 processor clears the CSRNEWDAT bit.

To accomplish this, write $0 \times 160$ foo to the CSR. This chooses start mode 1 , stop mode 0 , sets CSRUA (so that the same memory buffer will be used in each DMA transfer), sets CSRCNTRWE so that the TR register value will be written during the DMA transfer, and sets the values of the P and PCNTRL fields assuming that all of the input data buses will be in use all of the time.

Note that the CSR register contains a set of bits which specifies the board function but does not reflect daughter board status. Therefore, these bits can be typically set only once and reprogramming is not necessary for each DMA cycle.

5. Set the CSR2 to $0 \times 84$ to trigger the external data source and set the CSRHOLDENA bit.

6. Wait for the new data. It may take some time for the data to be acquired, so the processor can go ahead and do something else while waiting for the data. When ready to test for the completion of the DMA write cycle, one of the methods described in Subsection 2.8 can be used. Typically, the processor polls the CSRNEWDAT bit.

7. Do the data processing.

8. Set the CSR2 to $0 \times 84$ to trigger the external data source again. Note that this operation both clears CSRNEWDAT and sets CSRSAMPDC so that only one access to the FIFO memory daughter board registers is required in order to set up for each subsequent data acquisition and DMA transfer.

9. Continue to loop through steps 6,7 , and 8 until finished.

\subsection{A Typical Data Reduction Application}

In this type of application the input data can be buffered in the FIFO memory and/or in a SuperCard-2 memory buffer until needed. The assumption here is that the data reduction application takes as input a fixed number of data samples and analyzes that batch of data while the next batch of data is acquired. In this example, we also assume that the data reduction algorithm is too slow to execute in the time required to acquire a batch of data of the required size, but is fast enough to execute in less time than is required to acquire two batches of data. Thus, the data reduction application can only analyze every other batch of data. A second processor could be used to acquire and analyze the data that are skipped. Pass through mode will be used here to skip the unwanted batch of data. 
Since the data processing does not need to be coupled as tightly to the time of data acquisition as would be required in a feedback control application, start mode 2 is used here and the FIFO memory is used to buffer the data for awhile. Recall that in start mode 2 a DMA transfer cycle is triggered when the FIFO mernory becomes more than half full.

The size of a batch of data is well known. Therefore, we use stop mode 3 . In this mode, the sample counter is programmed so that the CSRNEWDAT bit is set after the exact number of data values required is acquired. Stop mode 3 allows, however, the DMA transfer operation to stop temporarily when the FIFO memory is empty so that the access of the 1860 processor to the SuperCard-2 memory is not blocked when there are no data available to be transferred.

In this application, we assume that there is an external trigger that starts the transfer of data from the external source to the FIFO memory at regular intervals, but that this trigger does not begin until after the daughter board is programmed properly for the first data acquisition cycle.

With this combination of start and stop modes, and with data being copied to the FIFO memory continuously, each time the FIFO memory becomes half full a DMA transfer is triggered. The DMA transfer continues until the FIFO memory is empty. Then the DMA transfer stops and the daughter board waits until the FIFO memory is again half full, at which time another DMA transfer starts. In this application, the CSRUA bit is clear so that each new DMA transfer starts writing data at the place that the previous DMA transfer left off. This process continues until the sample counter is incremented once past the value $2^{24}-1$ at which time the DMA transfer in progress stops, the TR register is copied to the SuperCard-2 memory (if CSRCNTRWE is set) and CSRNEWDAT is set. Here, CSRHOLDENA is also set so even if the FIFO memory reaches half full soon after the end of the last DMA transfer, a new DMA transfer will not begin (the incoming data will be buffered in the FIFO memory) until the i860 processor clears CSRNEWDAT.

A data acquisition and reduction program would proceed as follows.

1. Write 0 to all bits in the CSR register. This ensures that a DMA write operation cannot be triggered and that pass through mode is not chosen.

2. Reset the data acquisition circuits. Since the software cannot know the exact status of the data acquisition circuits, it is best to reset the system at the start of a data acquisition sequence. This reset will ensure that the FIFO memory is empty and the circuits are cleared of any anomalous condition that might have existed before the start of the program.

To reset the FIFO memory daughter board and the external data source, write 0x9 (where $0 x$ indicates hexadecimal) to the CSR2 register in order to set the CSRRESET bit and the CSREXTRESET bit. Then write 0x0 to the CSR2 register to end the reset state. 
When the CSRRESET bit is 1 , the values of the other CSR2 bits are not important.

3. Write the $A D$ register. The content of the $A D$ register is unknown otherwise because this register cannot be read. Therefore, the address of the DMA buffer should be written to the $A D$ register at the start of the data acquisition program. This value will not change until the register is written again.

4. Write 0xe20100 to the CSR register. This chooses start mode 2, stop mode 3, clears CSRUA so that as the DMA transfers restart each time the FIFO memory is half full, the data are written starting at the end of the buffer used in the previous DMA transfer, sets CSRCNTRWE so that the TR register value will be written at the end of the DMA transfer, and sets the values of the P and PCNTRL fields assuming that all of the input data buses will be in use all of the time.

The CSRPT bit is not set this time because we want to acquire the first set of data.

5. For this example, we assume that a batch of data consists of 2048 data values. Because two data values are written to memory on every transfer, the sample counter should be programmed so that the DMA transfer stops after 1024 data transfers. The actual value in the sample counter should be $2^{24}-1024$ which is 0xffec00. Set the CSR2 to Oxffrc0080 to set the CSRHOLDENA bit and the sample counter.

6. Now we wait for the data to arrive. Here we assume that the first batch of data does not start to arrive until the daughter board has been initialized as described in the previous few steps. We assume that at the end of the initialization, the FIFO memory is empty and the daughter board is waiting for data to arrive.

The i860 processor can poll the CSRNEWDAT in order to detect the completion of the DMA transfer of 2048 data values.

7. Before processing the newly acquired data, set up to receive the next batch of data, but since this data will not be processed, set up for pass through mode.

(a) Write $0 \times 0$ to the CSR register so that a data transfer cannot start while the setup is completed.

(b) No data will be written to the SuperCard-2 memory in this operation so the value in the address counter does not matter.

(c) Write 0x0xfffe0080 to the CSR2 register to set the CSRHOLDENA bit and the sample counter and clear CSRNEWDAT.

(d) Write 0xa80100 to the CSR register to choose start mode 2, stop mode 2 and pass through mode. Since the access to the SuperCard-2 memory by the i860 processor will not be blocked during these DMA operations, stop mode 3 is not necessary. Note that the CSR register with CSRPT set is written last because access to the other registers is limited when CSRPT is set. 
8. While the "ignored" batch of data is collected, process the batch of data that is already in the SuperCard-2 memory.

9. During the processing of the data, it is necessary to detect the end of the pass through mode DMA transfer and set up for the acquisition of the next batch of data which is a batch that will be processed. There are two ways to do this.

(a) In a convenient loop in the data processing algorithm, check the value of CSRNEWDAT periodically. When CSRNEWDAT is set, call a routine to set up to acquire the next set of data as described below.

(b) Set up an interrupt service routine to detect the end of the "ignored data" DMA transfer and set up to acquire the next batch of data. This is probably the most efficient method, but at this writing it has not been established how to do this.

10. To set up to acquire the next batch of data to be processed, proceed as follows. Here we assume that CSRNEWDAT and CSRHOLDENA are set so that a new data transfer cannot start until we are ready.

(a) Write 0xe20100 to the CSR register to choose start mode 2, stop mode 3, clear CSRUA and CSRPT and set CSRCNTRWE.

(b) Write the address of a second buffer in the SuperCard-2 memory to the AD register. This buffer is needed to hold the new data since the previous set of data that was acquired is still being processed.

(c) Write 0x0xfffe0080 to the CSR2 register to set the CSRHOLDENA bit and the sample counter and clear CSRNEWDAT. Clearing CSRNEWDAT allows the next DMA transfer to be triggered when the FIFO memory reaches the half full level.

11. Finish processing the current set of data.

12. Wait for the next set of data to be available. Then loop back to step 7 to set up to acquire the "ignored" batch of data and to process the new set of data.

13. Continue looping through this steps 7 through 12 until the desired amount of data has been acquired.

14. At the end of the data acquisition procedure write 0x0 to the CSR so that no DMA transfers can start.

\subsection{A Near Real Time Analysis Application}

In a "near real time" application, the analysis procedure is too slow to be executed in real time. Instead, the SuperCard-2 memory is used to hold as much data as possible and, when data acquisition is over, the data are analyzed. In this case, continuous memory mode is useful because the amount of data that can be placed in the SuperCard-2 memory is doubled. 
In this example, we assume that the situation is the same as in the previous section: a continuous, external trigger starts transfers of data into the FIFO memory. Start mode 2 is used again, but in this case there is only one large batch of data to be acquired and the processor does not need to access memory while the data are acquired, so stop mode 2 is used. In this example we assume that the entire SuperCard-2 memory will be filled with data. Assuming that the 16 Mbyte version of the SuperCard-2 is in use, there are 15.5 Mbytes available for data storage. This will hold $15.5 / 2$ Msamples so the sample counter should be set to acquire (15.5/2 Msamples)/2.

Proceed as follows.

1. Write 0 to all bits in the CSR register. This ensures that a DMA write operation cannot be triggered and that pass through mode is not chosen.

2. Reset the data acquisition circuits. Since the software cannot know the exact status of the data acquisition circuits, it is best to reset the system at the start of a data acquisition sequence. This reset will ensure that the FIFO memory is empty and the circuits are cleared of any anomalous condition that might have existed before the start of the program.

To reset the FIF.) memory daughter board and the external data source, write 0x9 (where $0 x$ indicates hexadecimal) to the CSR2 register in order to set the CSRRESET bit and the CSREXTRESET bit. Then write 0x0 to the CSR2 register to end the reset state.

When the CSRRESET bit is 1, the values of the other CSR2 bits are not important.

3. Write the $A D$ register. Since we are filling the SuperCard-2 memory, the $A D$ register should be written with $0 \times 0$.

4. Write 0xa40100 to the CSR register. This chooses start mode 2, stop mode 2, sets CSRUA (the value really doesn't matter here), clears CSRCNTRWE because there will be no room left in the memory to hold the TR register value, and sets the values of the $P$ and PCNTRL fields assuming that all of the input data buses will be in use all of the time.

5. The sample counter should be loaded with $2^{24}-15.5 \times 1024^{2} / 2 / 2$ which is $0 \times 20000$. So, write 0xc20000c0 to the CSR2 register. This sets CSRHOLDENA so that all DMA transfers will be disabled once the SuperCard-2 memory is full and sets CSRCM to choose continuous memory mode.

6. Poll CSRNEUDAT to detect the end of the DMA transfer. 


\section{Installation}

The FIFO memory daughter board is connected directly to the SuperCard-2 circuit board via two 100 pin connectors and is held in place with two alignment screws and five captured screws. This is illustrated in Fig. 6. The FIFO memory daughter board fills the VME slot on the right-hand side of the SuperCard-2. Note that this is unlike other daughter boards for the SuperCard-2 which, in general, occupy the same VME slot as the SuperCard-2 circuit board.

Before attaching the daughter board to the SuperCard-2, follow the CSPI instructions for installation of the SuperCard-2. Proper operation of the SuperCard-2 should be tested first, without the daughter board in place. Be sure to set the SuperCard-2 address switch before attaching the daughter board because the switch will not be accessible with the daughter board attached.

The daughter board uses 16 single-in-line resistor packages to provide the termination for the input signals that come from the digital data source. These are located in sockets at the front of the circuit board near connectors J6, J7, and J8 (Fig. 6). If only one daughter board is to be connected to the digital data source, then the black terminating resistor packages should be installed. If more than one daughter board is to be connected to the digital data source, then only the last daughter board connected to the input data cable should have the black terminating resistor packages installed. The other daughter boards on the cable should have the yellow resistor parkages installed in the terminating resistor sockets. The yellow packages simply provide a high impedance pull-up resistor to protect against damage from static electricity but do not provide transmission line termination.

To attach the daughter board to the SuperCard-2 do the following.

1. First it is necessary to install the alignment screws and some stand-off extensions on the SuperCard-2 board.

(a) Figure 6 shows the locations on the SuperCard-2 for the two alignment screws. In each hole, insert a screw from the non-component side of the circuit board and secure it in place with the threaded standoff.

(b) On the SuperCard-2 board there are five short stand-offs which must be lengthened. Screw a stand-off extension securely into each of the five stand-offs on the SuperCard-2. Use a pair of pliers to tighten the standoff slightly beyond finger-tight. 


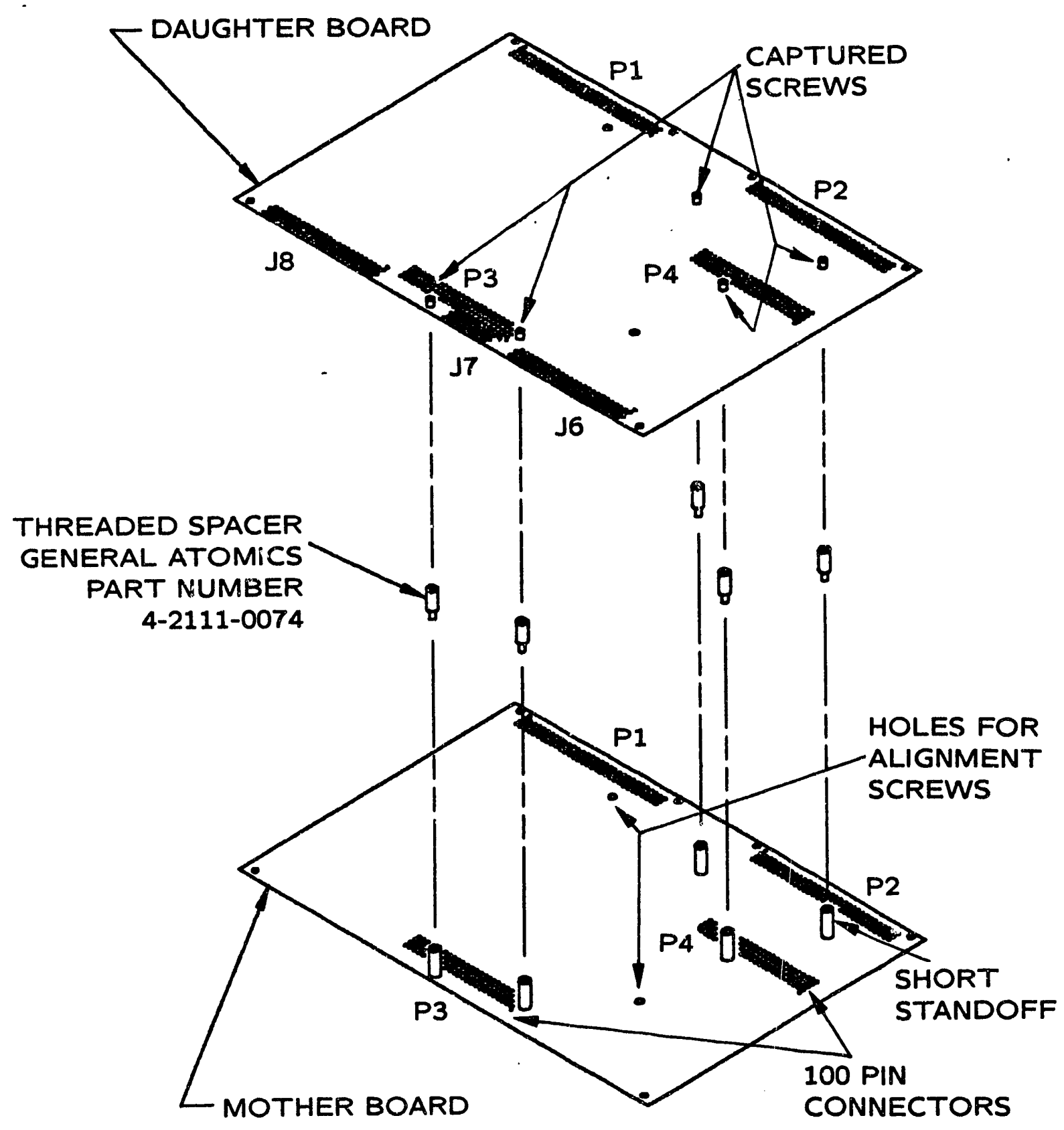

Fig. 6. Attaching the FIFO memory daughter board to the SuperCard-2. 
2. Lay the SuperCard-2 flat on a table and carefully lower the daughter board onto the SuperCard-2 with the two 100 pin connectors (Fig. 6) pointed down. Align the daughter board and the SuperCard-2 by ensuring that the two alignment screws on the SuperCard-2 pass through the corresponding holes on the daughter board.

3. As the alignment screws begin to pass through the holes on the daughter board, look between the two boards to be sure that the 100 pin connectors are aligned properly with the 100 pin sockets on the SuperCard-2. Use care to be sure that none of the pins on the 100 pin connectors become bent. Be gentle, no excessive force should be necessary.

4. Screw the five captured screws on the daughter board into the five stand-offs on the SuperCard-2 to draw the two boards together. Alternate between the screws, tightening each screw less than a turn each time. Try to draw the two boards together gradually while keeping the boards approximately parallel. Tighten each of the five screws securely.

5. Finally, put a nut on each of the alignment screws and tighten it.

When installing the two board set into the VME card cage, be sure to put equal pressure on each of the two boards so that they are inserted into the card cage together and so that the connections between the two boards are not stressed.

The VME slot containing the SuperCard-2 should have the interrupt chain and bus grant chain jumpers on the VME backplane removed as specified by CSPI. The VME slot containing the daughter board should have these jumpers in place.

To remove the two board set from the VME card cage, use the VME card extractor handles. Be sure to pull simultaneously on the top and bottom handles on both cards.

To remove the daughter board from the SuperCard-2 follow this procedure.

1. Remove the nuts from the two alignment screws.

2. Carefully unscrew the captured daughter board screws. These screws are used to disconnect the two 100 pin connectors, so you should alternate between the five screws, turning each screw a turn or less each time so that the daughter board and SuperCard-2 remain parallel during the extraction process.

3. Lift the daughter board off the alignment screws.

4. When the daughter board is not connected to the SuperCard-2, be sure to store it so that the two 100 pin connectors are not damaged.

The daughter board connects to the digital data source through ribbon cables that are attached to the three front panel connectors (labeled J6, J7, and J8 in Fig. 6). Refer to the digital data source manual for detailed installation information. 


\section{Acknowledgments}

The author would like to acknowledge the very valuable assistance in this project of E. Brown, G. Campbell, J. Land, E. McKee, A. Nerem, G. Phelps, C. Parker, J. Phillips, and T. Smith.

This is a report of work sponsored by the U.S. Department of Energy under Contract No. DE-AC03-89ER51114. 

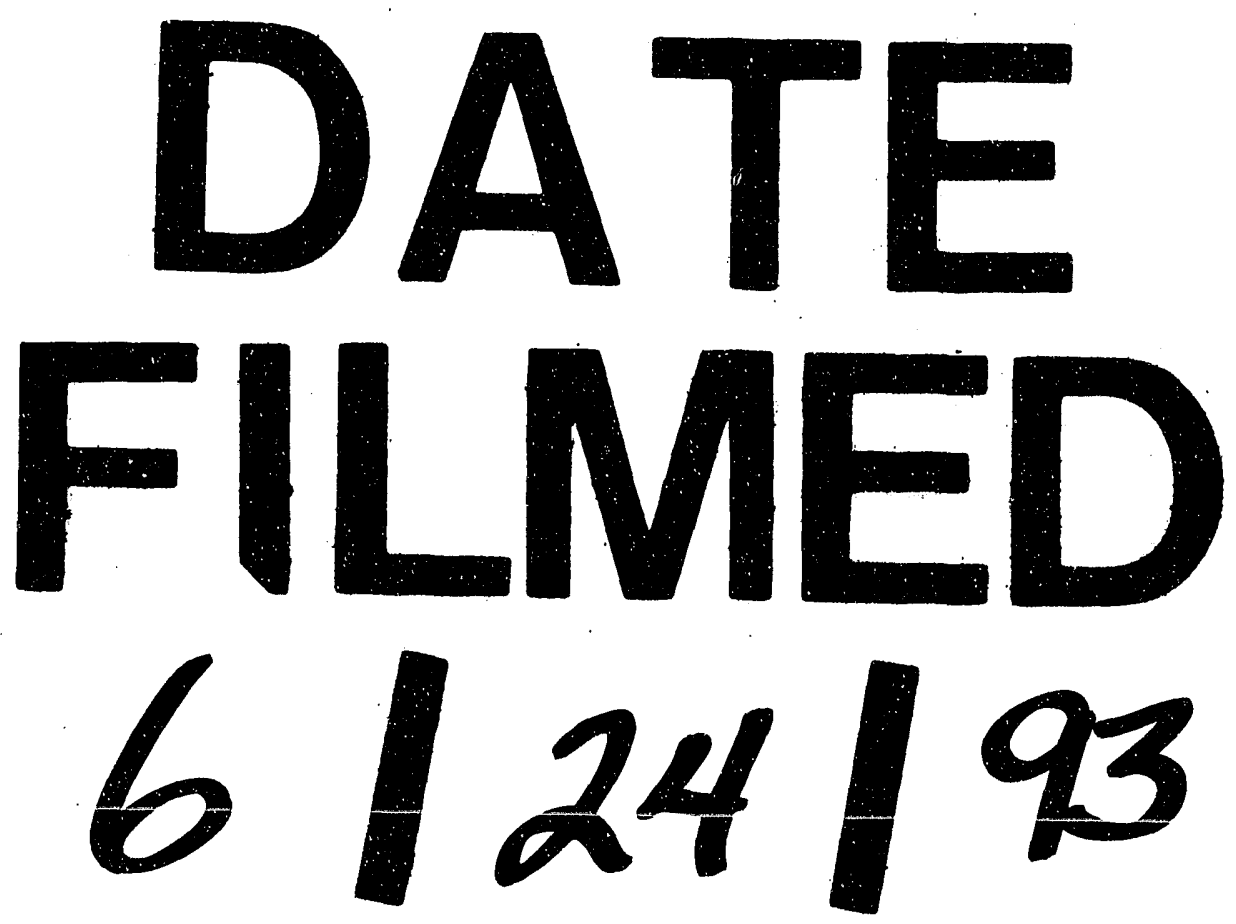
